\title{
GeoSail: An Elegant Solar Sail Demonstration Mission
}

\author{
Malcolm Macdonald, ${ }^{i}$ \\ SciSys Ltd., Bristol, BS4 5SS, U.K. \\ Gareth W. Hughes, ${ }^{i i}$ \\ EADS Astrium, Stevenage, SG1 2AS, U.K. \\ Colin McInnes, ${ }^{i i i}$ \\ University of Strathclyde, Glasgow, G1 1XJ, Scotland. \\ Aleksander Lyngvi, ${ }^{\text {iv }}$ Peter Falkner, ${ }^{4}$ Alessandro Atzei, ${ }^{4}$ \\ European Space Agency, Postbus 299, 2200 AG Noordwijk, The Netherlands.
}

\begin{abstract}
In this paper a solar sail magnetotail mission concept was examined. The $43 \mathrm{~m}$ square solar sail is used to provide the required propulsion for continuous Sun-synchronous apse-line precession. The main driver in this mission was found to be the reduction of launch mass and mission cost while enabling a nominal duration of 2 years within the framework of a demonstration mission. It was found that the
\end{abstract}

i Work conducted at University of Glasgow: now at SciSys Ltd., Malcolm.Macdonald@scisys.co.uk

ii Work conducted at University of Glasgow: now at EADS Astrium Ltd., Gareth.Hughes@astrium.eads.net

iii Professor, Department of Mechanical Engineering, Member AIAA colin.mcinnes@strath.ac.uk

iv Science Payload and Advanced Concepts Office, ESTEC alyngvi@rssd.esa.int 
mission concept provided an excellent solar sail technology demonstration option. The baseline science objectives and engineering goals have been addressed, and mission analysis for solar sail, electric, and chemical propulsion performed. Detailed subsystems have been defined for each propulsion system and it was found that the optimum propulsion system is solar sailing. A detailed trade-off as to the effect of spacecraft and sail technology levels, and requirements, on sail size has been presented for the first time. The effect of, for example, data acquisition rate and RF output power on sail size has been presented, where it was found that neither have a significant effect. The key sail technology requirements have been identified through a parametric analysis.

\section{Introduction}

The GeoSail mission concept is motivated by the desire to achieve long residence times in the Earth's magnetotail, enabling high resolution statistical characterisation of the plasma in a region subject to a variety of external solar wind conditions. ${ }^{1-4}$ This is accomplished by the novel application of a solar sail propulsion system to precess an elliptical Earth-centred orbit at a rate designed to match the rotation of the geomagnetic tail, the orientation of which is governed by the Sun-Earth line. Conventional missions to study the magnetosphere, such as Geotail, ${ }^{\mathrm{i}}$ Wind, ${ }^{\mathrm{ii}}$ Double Star ${ }^{\mathrm{iii}}$ and Cluster II $^{\text {iv }}$ are limited to inertial keplerian orbits, or investigation of the deep tail beyond the lunar distance. An inertially fixed orbit with an apogee inside the geomagnetic tail and interior to the lunar orbit will provide less than three months of science data from a single spacecraft due to the rotation of the see http://www.stp.isas.jaxa.jp/geotail/ or http://www-spof.gsfc.nasa.gov/istp/geotail/ see http://www-spof.gsfc.nasa.gov/istp/wind/ see $\underline{\text { http://sci.esa.int/science-e/www/area/index.cfm?fareaid }=70}$ see $\underline{\text { http://sci.esa.int/science-e/www/area/index.cfm?fareaid=8 }}$ 
geomagnetic tail with the Sun-Earth line. An appropriate sail steering strategy can however enable Sun-synchronous apse-line precession within the lunar radius, so that long-term residence is maintained within the magnetosphere by a single spacecraft.

A detailed assessment of competing propulsion methods; solar sailing, solar electric propulsion (SEP) and chemical propulsion will be presented within this paper, showing that solar sailing outperforms the other propulsion systems. The main driver in the investigation of the Sun-synchronous apse-line precession missions will be to reduce launch mass and mission costs, while enabling a nominal operational period of 2 years within the framework of a demonstration mission. Note that a detailed subsystem budget is defined for each propulsion system. It will be shown that a solar sail technology demonstration mission, within the possible framework of SMART (Small Missions for Advanced Research and Technology) enables a useful science return for at least 2 years of residence within the magnetotail, delivered using a low-cost Vega launcher. The GeoSail mission detailed within this report would provide a key path towards future large solar sail missions, such as Solar Polar Orbiter (SPO) and Interstellar Heliopause Probe (IHP). 5 - 7

\section{Science Objectives}

The primary science goals of the GeoSail mission are to understand how spontaneous magnetic reconnection occurs in the magnetotail current plasma sheet, and to understand the mechanisms behind reconnection mode destabilisation and saturation in the magnetotail. In the compressed region of the magnetosphere between the Earth and the Bow Shock, GeoSail will attempt to understand the effects and the process of reconnection and particle dynamics on the Sunward side of the Earth. In addition, measurements can investigate the boundary layer along the magnetopause. A suite of plasma, field and particle instruments will be used to achieve the science goals of the mission. An orbit perigee of 11 
Earth Radii will allow the spacecraft to pass along the magnetopause, and an apogee of 23 Earth Radii will allow measurements of the magnetic reconnection region in the magnetotail. This orbit, with a major axis permanently aligned with the magnetotail axis, will enable very high time resolution measurements of the magnetosphere. ${ }^{8}$

\section{Engineering Goals}

This study envisages GeoSail within the potential framework of a future SMART. Thus, solar sail technology demonstration is the primary mission goal, with the science goals outlined above as secondary mission goals. Mission success will only require that the engineering goals are met, however it is highly desirable that science data also be acquired. The mission concept must provide heritage towards future solar sail missions and as such sail boom and film stowage, packing and deployment must be designed to allow heritage and extrapolation to larger sail missions, such as SPO. Packing and integration into the launch vehicle has been identified as a key technology issue for future, large sail missions. ${ }^{5}$ The deployment of a fully functional solar sail must be demonstrated in a real space environment; GeoSail will perform this demonstration. During sail deployment and later during operations, observation and measurement of the sail dynamics must be performed. This will be performed by instrumentation on-board the sail, such as load sensors, and simultaneously by cameras on-board the kick-stage, which will have a limited formation flying capability to allow visual documentation of sail deployment and early operations. During sail deployment we must observe and measure:

- Management and behaviour of the packaged film during deployment.

- Controlled release of film and booms.

- Film tension, boom loading and structural characteristics. 
Following sail deployment, GeoSail must demonstrate the stability, controllability and operational capability of a solar sail, through:

- Demonstration of measurable propulsive performance (apse-line rotation).

- Verification of attitude control models, concepts and operations.

- Demonstration of sufficient sail slew rate capability for future missions with no, or negligible, propellant expenditure.

- Verification of trajectory control algorithms.

Furthermore, test-ground model validation must be performed, such as to confirm predicted structural frequencies, sail performance degradation and sail force models (at a range of sail pitch angles). The navigation and guidance of a sail must also be demonstrated. The measurement of parameters which are difficult to measure / predict on the ground must be performed, such as:

- Analysis of response to thermal environment.

- Attitude perturbations due to uncontrollable variations in sail shape and performance degradation.

- Actual sail performance, resulting from actual sail shape and optical surface performance degradation.

- Actual sail pointing / stability capability.

In addition to the above sail deployment and control goals, measurement and analysis must be performed as to the effect of the sail on the local space environment. This is a key mission goal. The effect of the sail on the local plasma environment must be measured and quantified and then compared with prior ground analysis predictions. The effect of the sail on the local space environment is key to many future sail missions, such as Geostorm. ${ }^{9}$ It must be determined if a science suite can operate while attached to a solar sail, or whether future sail missions are limited to scenarios where the sail is jettisoned prior to mission operations, such as SPO and IHP. The final engineering mission goal of 
GeoSail is the successful demonstration of a sail jettison and separation manoeuvre. This is a key mission stage in future missions such as SPO and IHP where the core mission can only begin once the sail has been jettisoned and a safe sail separation manoeuvre performed.

\section{Mission Analysis}

The GeoSail orbit designed to achieve the stated science goals has a perigee located above the planetary dayside at approximately 11 Earth radii $\left(\mathrm{R}_{\mathrm{E}}\right)$, corresponding to alignment with the magnetopause. Apogee is aligned with the geomagnetic tail reconnection region on the night-side of the Earth, at $23 \mathrm{R}_{\mathrm{E}}$. The orbit plane is within the ecliptic plane. A key feature of the GeoSail orbit is the ability to investigate the near-downstream region over an extended period. Conventional missions have achieved extended observation times only in the deep tail by executing double-Lunar flybys to precess the orbit apse-line. The utilisation of a small solar sail allows orbit apse-line precession without the requirement of going as far as the Moon, at approximately $60 \mathrm{R}_{\mathrm{E}}$, thus enabling extended study of this key region of the near-tail. The GeoSail orbit orientation is illustrated in Figure 1, where we see that the Sun-Earth line is coincident with the orbit major axis. We note immediately from Figure 1 that the spacecraft will experience a prolonged Earth shadow event every apogee due to the required orbit orientation. 


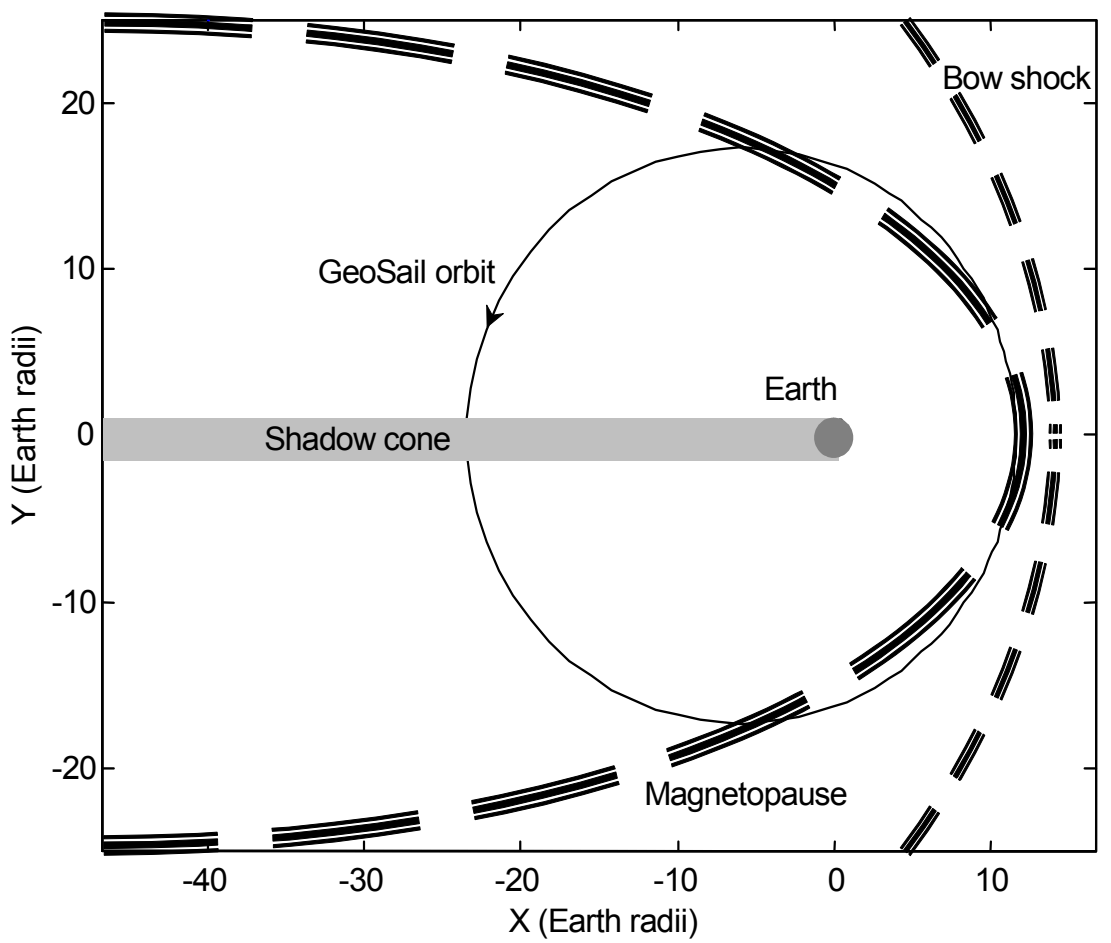

Figure 1 GeoSail orbit illustration in rotating reference frame, with magnetosphere detailed

As the spacecraft orbits within the ecliptic plane and the sail normal is assumed to be directed within the ecliptic plane, such that there will only be an in-plane perturbing acceleration due to solar radiation pressure, only three osculating orbital elements are required to describe the evolution of the resulting trajectory. An analytical analysis of the GeoSail orbit has previously been undertaken and can be found in Ref. 2. Within Ref. 2 a simple Sun-pointing steering law is derived and shown to induce an independent secular variation in the argument of pericentre, thus by varying the sail thrust magnitude the rate of change of argument of pericentre can be varied. The required sail characteristic acceleration is found to be $0.09985 \mathrm{~mm} \mathrm{~s}^{-2}$. The characteristic acceleration is defined as the acceleration experienced by an idealised sail at a heliocentric distance of 1 astronomical unit (AU) while the sail normal is directed along the Sun-line in the anti-Sun direction. ${ }^{10}$ Note the defined sail characteristic acceleration is adjusted to account for the prolonged shadow event each orbit. ${ }^{2}$ Furthermore, we note 
that as both the Sun-line rotation rate and the solar radiation pressure vary as the inverse-square of the planetary heliocentric distance, the forced precession of the elliptical orbit apse-line and the required rate of change have the same functional relationship. Thus, the simple Sun pointing steering law maintains a Sun-synchronous precession of the elliptical orbit apse-line, even if the planetary orbit is noncircular.

\section{Solar Sail Orbit Evolution}

The GeoSail orbit was modelled using modified equinoctial elements as the equations of motion, which are propagated using an explicit, variable step size Runge-Kutta $(4,5)$ formula, the Dormand-Price pair, ${ }^{11}$ with relative and absolute error tolerances of $10^{-8}$ ensuring minimal truncation error. The Sun is modelled as a uniformly bright, finite disk, while the sail acceleration is corrected to account for the true solar distance. Third body gravity perturbations due to both the Moon and the Sun are modelled as point masses, while the Earth's gravity is modelled using an $18^{\text {th }}$ order Geopotential model of zonal and tesseral harmonics, the Earth Gravity Model 1996. Lunar and Terrestrial shadow events are modelled and distinguish between umbra and penumbra. The variable step size integrator typically takes over 2500 steps per orbit, or an average step size of approximately five minutes.

The GeoSail trajectory propagation results are shown in Figure 2 and Figure 3, for a start epoch of 3 January 2010. We note that the orbit propagation analysis assumes the sail thrust is always pointing at exactly zero pitch to the sun-line and is always along the sail normal vector. Future analysis should consider the effect of sail pointing stability.

We note from Figure 2 that the orbit elements have both short and long period oscillations. The short period oscillation corresponds to the orbit period, while the long period oscillation corresponds to the 
period of the Earth about the Sun. The short period oscillation has negligible secular variation over the orbit period. However, the long period oscillation results in perigee and apogee variations of approximately one Earth radii from the nominal value. It was found that by varying the propagation start epoch through the Earth's orbit we could reverse the variations seen in Figure 2, such that perigee radius would vary up to 12 Earth radii and apogee down to 22 Earth radii.

Figure 3 shows the variation of the orbit plane from the ecliptic plane, which is mainly due to lunar gravity and to a lesser degree solar gravity. It was found once again that varying the propagation start epoch and thus the initial position of the sail with respect to the Moon could alter this variation. We also see in Figure 3 the variation of the orbit major axis from the Sun - Earth line. It is found that the major axis varies by up to $7 \mathrm{deg}$ from the Sun - Earth line in the core mission duration of two years. The orbit variations shown in Figure 2 and Figure 3 from the nominal GeoSail orbit should have minimal impact on science returns and may even have a positive impact. The magnetosphere is a dynamic region of space and the exact location of the key regions of interest vary with solar conditions, thus a dynamic orbit should allow for some of these variations to be offset and increase the chance of the spacecraft passing directly through the regions of interest.

We note that the $3^{\text {rd }}$ body gravity perturbation magnitude can peak at up to $0.09 \mathrm{~mm} \mathrm{~s}{ }^{-2}$, when the sail Moon distance is minimum; thus at peak lunar perturbation the disturbing acceleration is approximately 0.91 times the sail acceleration. The Earth shadow events were found to have a typical duration of 200 minutes, while the lunar shadow events in this trajectory are no more than 90 minutes. It is found on analysis of the data that lunar and Earth shadow events are always more than 24 hours apart, thus allowing sufficient time for systems recovery after one shadow event, prior to the next. Note the systems should be able to survive two Earth shadow events with no recovery between them in the event of an emergency mode. Furthermore, it was found that by varying the start epoch by only a few days 
from almost any given epoch a two year trajectory can be found that has very few, or even no lunar shadow events. Similarly, it was found that a sub-optimal epoch can result in very long lunar shadow events and as such the sail deployment date must be carefully considered to ensure against prolonged lunar shadow events of duration greater than the systems design value. Note that such a requirement assumes an accurate sail thrust model is available in order to precisely propagate the sail trajectory.
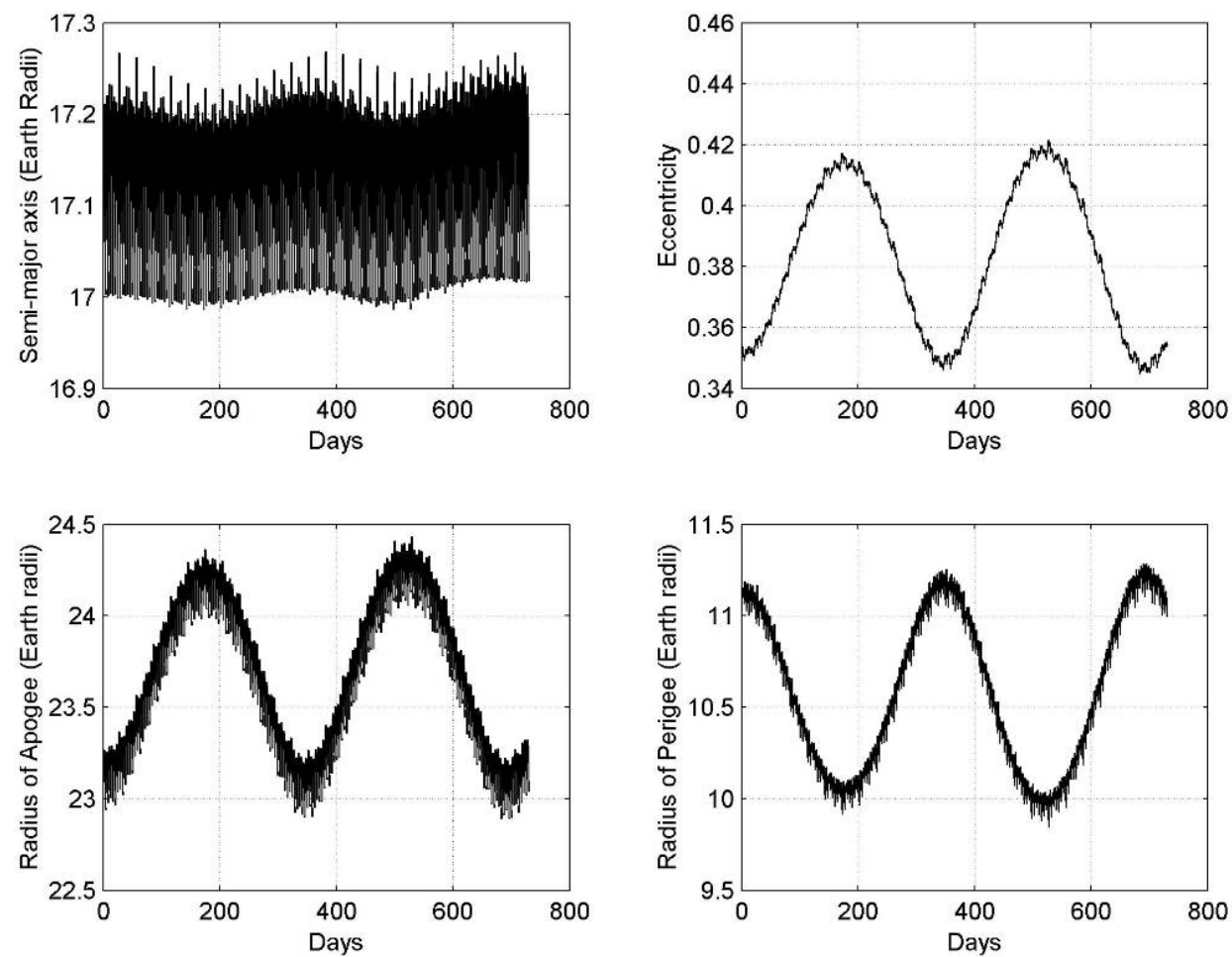

Figure 2 GeoSail orbit propagation over 2 years. 


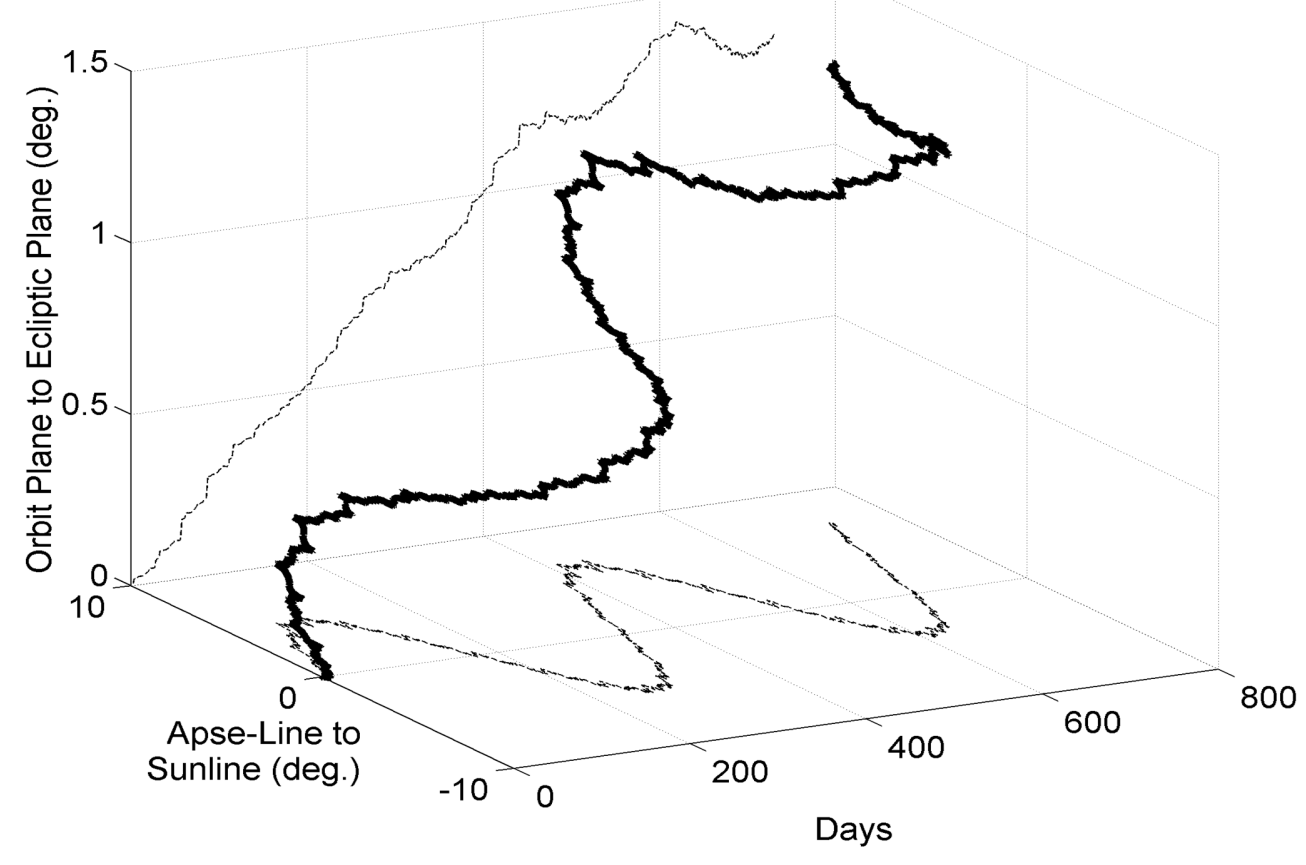

Figure 3 Angle of apse-line from the Sun-line and angle between orbit plane and Ecliptic plane, both against time and both projected into the axis.

\section{Electric Propulsion Analysis}

To compare competing propulsion methods with solar sailing, an analysis of Sun-synchronous apseline precession was performed for ion propulsion, specifically SEP. The required thrust, defined by the required acceleration for apse-line precession, will be used to calculate the required power level to size the solar arrays for a GeoSEP mission later in this paper.

Several SEP steering strategies were considered. However, it is clear that if the required rate of change of argument of pericentre is fixed, as is the orbit size, shape and orientation, then the propellant mass fraction is minimised through use of a locally optimal variation of argument of pericentre control law. ${ }^{10-13}$ The symmetry of the thrust profile produced by the locally optimal variation of argument of pericentre control law means that it complies with the conditions defined previously to produce an independent secular variation in the argument of pericentre. ${ }^{14,15}$ The mean required acceleration from 
the SEP system was found to be $0.092 \mathrm{~mm} \mathrm{~s}^{-2}$. Note however that the actual required acceleration will vary through Earth's orbit, such as to match the variation in the required rate of change of the apse-line. It is found that a one year SEP trajectory requires a propellant mass fraction of 0.1518 .

The Qinetiq T5 engine has a possible lifetime of 10000 hours at $25 \mathrm{mN}$ thrust level, and can be throttled smoothly over a wide range. ${ }^{16}$ The larger $25 \mathrm{~cm}$ Qinetiq T6 can provide a thrust of up to 300 $\mathrm{mN},{ }^{17}$ which is unnecessarily high for the GeoSEP mission, so the T6 will not be discussed. The Qinetiq T5 is a likely candidate for the mission using SEP. A parametric analysis was performed using the Qinetiq T5 thruster with an empirical relationship used for thrust throttling. ${ }^{18}$ Both the specific impulse and beam conversion efficiency are a function of the instantaneous thrust required. The empirical relationships are curve-fits to the actual physical data, when the Qinetiq T5 thruster was subjected to a rigorous throttling test over the range 0 to $30 \mathrm{mN}$. The exhaust gas would usually need to be neutralised, especially for a geomagnetic tail mission, since the ionised particles would interfere with science measurements and spacecraft subsystems. The curve-fits used were those for a thruster with an exhaust gas neutraliser. Figure 4 shows the propellant mass as a function of dry spacecraft mass, not including the Xenon propellant tanks, for mission duration of 2 years. The mass of the tanks is also shown in this figure, assuming their mass is $16.3 \%$ of the propellant mass, as for the Deep Space 1 spacecraft. ${ }^{\mathrm{i}}$ It is seen that slightly more propellant is used when the neutraliser is included. Note that for a nominal 2 year mission, the relatively low thrust levels mean that the specific impulse is rather low, and so the propellant consumed is higher than may be expected.

see http://nmp.jpl.nasa.gov/ds 1/ 


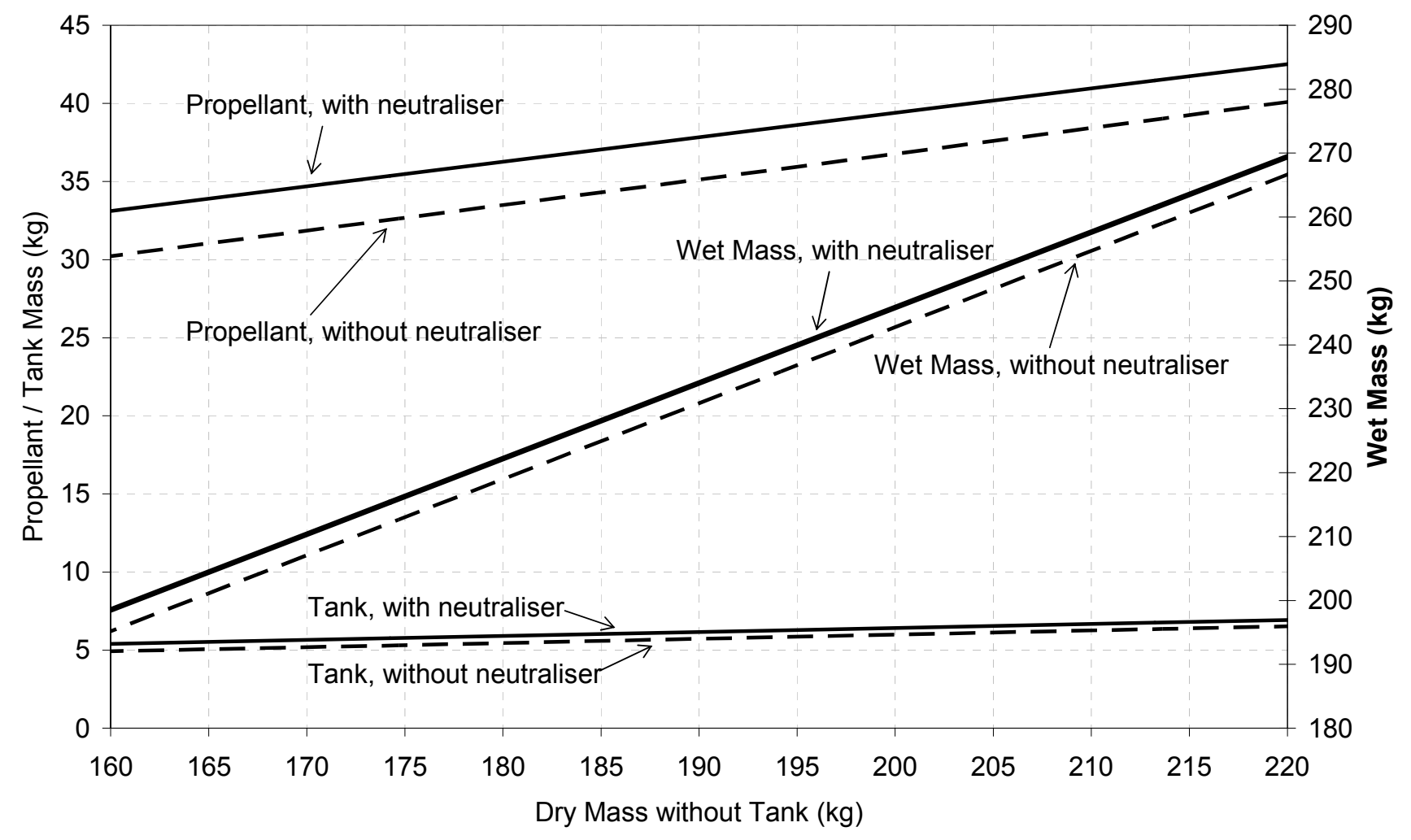

Figure 4 Qinetiq T5 (variable specific impulse and efficiency) propellant mass and representative tank mass, using empirical thrust relationship over 2 years.

\section{Chemical Propulsion Analysis}

Apse-line precession can be achieved using chemical propulsion via a 2 burn strategy, applying two equal impulses to connect the initial and rotated orbits through the arc of an intermediate transfer ellipse. Equating the change in the argument of perigee per orbit, with total burn $\Delta v$ to the desired change per orbit provides an approximate measure of the $\Delta v$ required for Sun-synchronous apse-line precession. In reality each burn will be finite in duration, of order $10-20$ seconds, which will lead to some finite burn losses, however these losses are neglected in this simplified analysis. The $\Delta v$ per orbit is $25.55 \mathrm{~m} \mathrm{~s}^{-1}$ for the $11 \times 23$ Earth radii orbit, which leads to a yearly $\Delta v$ requirement of $2273.4 \mathrm{~m} \mathrm{~s}^{-1}$. The propellant mass can thus be obtained using the rocket equation. Using a specific impulse of 350 seconds, representative of a liquid bi-propellant propulsion system, assuming that the tank mass is 
$10 \%$ of the propellant mass and the valves/pipes mass is $30 \%$ of the tank mass; for a dry mass (minus tank and pipes) of $100 \mathrm{~kg}$, the propellant mass is $440 \mathrm{~kg}$, meaning the initial wet mass is of order 600 $\mathrm{kg}$. This is clearly much larger than the solar sail and SEP spacecraft concepts. It seems that chemical propulsion would only be competitive for mission durations of less than 1 year.

\section{Launcher Selection}

Whichever launch vehicle is selected, an auxiliary upper stage will be required to obtain the final GeoSail operational orbit. Limiting our analysis to the Soyuz-2B and the Vega we can trade the potential orbit acquisition strategies. The Soyuz-2B, from Kourou, can launch direct into an ecliptic plane orbit of $6578 \times 146697 \mathrm{~km}$ (200 km altitude perigee and 23 Earth radii apogee), with a payload mass of $2220 \mathrm{~kg}$, thus only requiring a perigee raise manoeuvre to acquire the GeoSail orbit. The Vega is limited to Low Earth Orbit delivery, but at approximately half the cost of a Soyuz-2B. The anticipated maximum delivery altitude of the Vega to a circular orbit is $1500 \mathrm{~km}$, with a payload of $1525 \mathrm{~kg}$. Launch to a circular $1500 \mathrm{~km}$ orbit would thus require a minimum of two burns to acquire the GeoSail orbit and would thus eliminate the use of a single solid rocket motor.

\section{Soyuz-2B Orbit Acquisition}

Following delivery onto an ecliptic plane orbit of $6578 \times 146697 \mathrm{~km}$ we require only a single perigee raise manoeuvre at apogee to obtain the GeoSail operational orbit. The GeoSail orbit acquisition requirements for a Soyuz-2B launch, from Kourou, give a nominal insertion $\Delta v$ of $846 \mathrm{~ms}^{-1}$. We note that a further $65 \mathrm{~ms}^{-1}$ is included for correction manoeuvres which need not be performed by the primary motor. Either a solid or liquid motor could perform the perigee raise burn. 


\section{Vega Orbit Acquisition}

Following delivery onto an ecliptic plane circular orbit of $1500 \mathrm{~km}$ altitude we require a two-burn manoeuvre to obtain the GeoSail operational orbit. The GeoSail orbit acquisition requirements for a Vega launch are such that the nominal insertion $\Delta v$ is $3573.5 \mathrm{~ms}^{-1}$. We note that a further $268 \mathrm{~ms}^{-1}$ is included for correction manoeuvres which need not be performed by the primary motor. The GeoSail orbit acquisition manoeuvre requires a liquid motor and due to the high-energy requirements the motor will require to be a bi-propellant stage in-order to fit within the mass and volume launch capabilities of the Vega vehicle.

A crude cost comparison suggests that the GeoSail orbit acquisition motor following a Soyuz-2B launch will cost approximately half the cost of the kick-stage required following a Vega launch. However, the extra launch cost of the Soyuz vehicle means that if at all possible we would like to select the Vega vehicle. Selection of a Vega is on condition that it is possible to launch a large upper stage safely on the vehicle, that the cost of the upper stage, including an Engineering Analysis System to monitor sail deployment (as will be discussed later), is less than $\sim 10 \mathrm{M} €$ and that the volume and mass constraints of the Vega can be met, as was found to be the case by this assessment study.

\section{System Analysis and Configuration}

The system analysis is driven by the mission requirements as well as the general requirements and objectives of a demonstration mission. The system analysis is presented for the GeoSail mission as a solar sailing demonstration mission, which is later adapted for electric and chemical propulsion alternatives to enable a comprehensive propulsion trade. 
Delivery of the spacecraft to the GeoSail operational orbit requires a large $\Delta v$ capability from a dedicated transfer stage. Transfer from the launch vehicle to the GeoSail orbit using the solar sail is a prolonged process due to the low level of sail performance required for orbit procession, ${ }^{19}$ thus requiring the use of an auxiliary upper-stage, as previously detailed.

At all stages of the mission design the standard ESA/ESTEC assessment study margin philosophy is adopted unless otherwise stated. As such the design maturity margin is added at equipment level, where $>5 \%$ is added for off-the-shelf items (European Cooperation for Space Standardisation, ECSS, Category: A/B), > $10 \%$ for off-the-shelf items requiring minor modifications (ECSS Category: C) and $>20 \%$ is added for new design/development items, or items requiring major modifications or redesign (ECSS Category: D). The nominal on-orbit value is the current best estimate (CBE) plus the design maturity margin (DMM). A system level margin of $20 \%$ is added to mass and power to obtain the nominal launch value. The system level margin is not applied to any propellant residuals or additional propellant. The power systems are sized for the worst-case scenario, which in this case is end-of-life. Note the power system is designed for a realistic mode of operations and not simply with all systems active and drawing power. Data processing margins are $100 \%$ for science data and a minimum of $50 \%$ for engineering data, while on-board processor peak use shall not exceed $50 \%$ maximum capability. Communication links at all times maintains a minimum $3 \mathrm{~dB}$ margin in mean, favourable and adverse conditions to $3 \sigma$ confidence.

Throughout this paper full redundancy is maintained, except for the solar sail, science instrumentation, structure, propellant tanks, et cetera. Selection of full redundancy increases cost and volume; however it also provides the maximum demand on the sail technology, which is the primary study driver. Furthermore, the demonstration of new technology increases mission risk and as such redundancy is included as a risk reduction feature. 


\section{Spacecraft System Budgets}

Table 1 shows an overview of the GeoSail systems design. Note, the system design was conducted using a spreadsheet with optimisation and goal-seek capability. This allows the use of circularreferences to produce inter-dependences which reflect the real-world. For example, in the definition of the communication link budget a design slant range is required; if we increase this value the link margin will decrease, thus the goal-seek tool is used to enforce a minimum $3 \mathrm{~dB}$ margin by, for example, increasing the spacecraft RF power. Due to the spreadsheet design this then automatically impacts the power systems design load, which then due to the circular-references increases the required power due to, for example, increased thermal loads. Finally the in-built optimisation tools converge onto a stable design point for the new design slant range. The use of a spreadsheet with optimisation and goal-seek capability allows the system trades presented in this paper to be performed.

The spacecraft and solar sail are assumed at all times to be 3-axis stabilised (square sail), driven by the current solar sail technology focus on this architecture / attitude control method and not by the science which may in some scenarios prefer a spinning spacecraft. However, should the sail technology focus change in-light of SPO and IHP requirements to a spinning sail then GeoSail should demonstrate the required technology for these later missions. In this respect science is somewhat sacrificed in order to provide suitable heritage to future European sail missions. It was not considered within this study to either spin the spacecraft on a three-axis sail, or have a spinning spacecraft following sail jettison in the event of sail deployment failure. The masses presented in Table 1 are based on a main sail boom specific mass of $40 \mathrm{~g} \mathrm{~m}^{-1}$, a Teonex ${ }^{\circledR i}$ Polyethylene Naphthalate (PEN) sail film thickness of $3.5 \mu \mathrm{m}$ and sail front and rear coatings of $0.1 \mu \mathrm{m}$ Aluminium and $0.01 \mu \mathrm{m}$ Chromium, respectively. The sail system analysis will be presented later, quantifying the effect of varying these parameters through the

i Teonex is a registered trademark of E. I. du Pont de Nemours and Company. 
use of circular-references and optimisation tools within the spreadsheet in-order to explain the origin of the selected technology requirements. We note that although the sail is presented as a separate system it cannot operate independent of the spacecraft, as it is in-effect just another sub-system of the spacecraft.

\begin{tabular}{|c|c|c|c|}
\hline System & $\begin{array}{c}\text { CBE Mass } \\
(\mathrm{kg})\end{array}$ & $\begin{array}{l}\text { DMM } \\
(\%)\end{array}$ & $\begin{array}{c}\text { CBE Mass + DMM } \\
(\mathrm{kg})\end{array}$ \\
\hline Science Instruments & 11.5 & 10.0 & 12.7 \\
\hline Attitude \& Orbit Control System, AOCS (dry) & 10.5 & 5.0 & 11.0 \\
\hline Telemetry, Tracking and Command, TT\&C & 16.3 & 5.0 & 17.1 \\
\hline On-Board Data Handling, OBDH & 1.1 & 10.0 & 1.2 \\
\hline Thermal \& Radiation & 4.7 & 10.0 & 5.1 \\
\hline Power & 17.5 & 10.0 & 19.3 \\
\hline Mechanisms \& Structure & 17.1 & 7.2 & 18.5 \\
\hline Spacecraft Nominal Dry Mass At Launch & & & 84.7 \\
\hline AOCS propellant, inc. sail separation allowanc & and a margin & & 2.3 \\
\hline Spacecraft Nominal Wet Mass at Launch & & & 87.2 \\
\hline Solar Sail Nominal Mass at Launch (see also T & ible 5) & & 58.9 \\
\hline Nominal On-Orbit Mass & & & 146.1 \\
\hline Kick-stage mass (dry) (see also Table 3) & 192.7 & 5.2 & 202.7 \\
\hline Consumables & & & 837.4 \\
\hline Kick-stage mass (wet) & & & 1040.1 \\
\hline Nominal Launch Mass & & & 1186.1 \\
\hline ESA System Level Margin & & $20 \%$ & 237.23 \\
\hline \multicolumn{3}{|l|}{ Propellant Residual (at $1.5 \%$ of Propellant Mass) } & 12.6 \\
\hline \multicolumn{3}{|l|}{ Total Mass At Launch } & 1435.9 \\
\hline \multicolumn{3}{|l|}{ Vega launch capacity (to $1500 \mathrm{~km}$ circular orbit) } & 1525.0 \\
\hline \multicolumn{3}{|l|}{ Launch Margin } & $89.1 \quad(5.8 \%)$ \\
\hline
\end{tabular}

Table 1 GeoSail System overview 


\section{Science Instruments}

During this assessment study no detailed science instrument assessment was performed, rather the existing published GeoSail science suite was adopted. ${ }^{4}$ The current strawman payload comprises five instruments, detailed in Table 2, and is adapted from the NASA THEMIS mission. The science suite is highly representative of what a typical GeoSail science suite may look like and as such allows the system requirements of GeoSail to be identified.

The Science Data Rate Stream is the rate at which data is acquired from the science instruments, which is then buffered onboard the spacecraft, prior to downlink. The science data rate stream breakdown is detailed in Table 2. The required telemetry downlink rate is largely driven by the science suite requirements.

\begin{tabular}{crrrr} 
Mass Fraction & Routine & Particle burst & Wave burst Raw Data \\
(with 10\% DMM & Telemetry & Telemetry & Telemetry & Acquisition \\
on each instrument) & Rate (bps) & Rate (bps) & Rate (bps) & Rate (bps) \\
\hline $2 \%$ & 256.0 & 2048.0 & 8192.0 & 593.9 \\
$19 \%$ & 608.0 & 4395.0 & 4395.0 & 1062.4 \\
$9 \%$ & 512.0 & 1707.0 & 4096.0 & 703.2 \\
$18 \%$ & 555.0 & 5120.0 & 16384.0 & 1328.1 \\
$52 \%$ & 555.0 & 5120.0 & 16384.0 & 1328.1 \\
- & $88 \%$ & $10 \%$ & $2 \%$ & -
\end{tabular}

Table 2 Science data rate stream breakdown 


\section{Science Suite Integration}

It is anticipated, based on previous studies, that a solar sail will have pointing control of approximately $360-3600$ arcsecs. $^{20,21}$ Pointing knowledge of the science suite would be high as this can be determined by the spacecraft AOCS. Pointing stability of the science suite is difficult to determine, since we note that the lowest structural mode frequency of a solar sail is typically below $0.1 \mathrm{~Hz}^{20,21}$ It is therefore possible that the pointing stability over short timescales may be quite high. It appears therefore that sufficient stability, accuracy and knowledge can be obtained to match science suite requirements.

Prior analysis of the interaction between the sail and local space environment is limited, so definitive statements are unwise. Analysis of prior studies on the interactions between the sail and the local environment at $1 \mathrm{AU}$ do however allow some limited extrapolation. We note however that this work is preliminary and forms only the first step of a much larger research program that is commencing within NASA/JPL, and makes many considerable assumptions. ${ }^{22}$ Of importance here are plasma interaction effects due to the solar wind, which may impact on plasma analyser data. The solar wind can be considered fully ionised, electrically neutral, magnetised plasma flowing outward from the Sun. The basic characteristics of the solar wind flowing around a solar sail are thus the same as that of collisionless, mesothermal plasma flowing around an obstacle. Moreover, as both the ion gyroradius ( $400 \mathrm{~km})$ and the electron gyroradius $(\sim 1.5 \mathrm{~km})$ are much larger than the typical solar sail dimension $(<$ $50 \mathrm{~m}$ ), the solar wind flow may be considered as an unmagnetised, collisionless plasma flow for our problem. It was found in Ref. 22 that the sail was surrounded by a plasma sheath within which the potential is positive compared with the ambient plasma and followed by a separate plasma wake, which is negative relative to the plasma. This structure departs dramatically from a negatively charged plate such as might be found in the Earth's ionosphere on the night side where both the plate and its negative wake are contiguous. Furthermore, at $1 \mathrm{AU}$ the plasma sheath in the ram side starts at a distance of $\sim 2$ 
$\lambda_{D}$ (debye length). Notably, the sail size appears to have minimal impact on the plasma sheath, although the potential in the wake region is significantly different. Garrett and Wang, Ref. 22, concluded that although the plasma sheath at $1 \mathrm{AU}$ extends to a distance of perhaps as much as $50 \mathrm{~m}$ in front of the sail, its effects on the solar wind electron measurement made near the sail surface should be minimal. This is because the solar sail floating potential is only about $10 \mathrm{~V}$, while the solar wind electron temperature is $\sim 40 \mathrm{eV}$. However, the sheath may have some adverse effects on solar wind proton measurements made within the sheath. As such we can state that in order to make any meaningful plasma analysis while attached to the solar sail we would need to place the instruments perhaps several debye lengths ahead of the sail, or one or two debye lengths to the side, but definitely not behind. Determination of the debye length requires accurate knowledge of the plasma conditions, which vary widely. However, we can estimate the debye length to be perhaps $20 \mathrm{~m}$. Therefore, placement of instruments as much as several debye lengths ahead of the sail would require a separation distance of $>40-50 \mathrm{~m}$, an unrealistic prospect. Similarly, placement of instrumentation one or two debye lengths to the side of the sail would require a very large separation distance.

Consideration of the effect of the sail on the magnetometer environmental conditions is even more difficult to determine. However, the potential exists for the creation of a self-generated contamination cloud entrapped around the sail, with perhaps a small magnetosheath like a comet on the size of the largest dimensions of the sail. In conclusion, it is unclear whether the science suite can be suitably integrated into a solar sail mission. Significant technology development and analysis is required to validate any potential integration solutions, such as turning the sail to an edge-on profile to the solar wind to minimise solar particle drag profile and perhaps thus enable use of the science suite. Due to the difficulties of providing an adequately sterile instrument environment and the lack of knowledge of sail / space environment interaction and its impact on future sail missions, a key mission goal of GeoSail is to measure the sail / space environment interaction to enable enhanced mission planning of 
future sail missions. Measuring such an interaction could in principle be performed in several ways, for example, if the instruments were active prior to sail deployment then the before and after measurements could be compared. Similarly, by jettisoning the sail the acquired results could be compared. Furthermore, if an instruments location could be varied with respect to the sail then the sail / space environment interaction could be better quantified. This would perhaps be best performed simultaneously at varying locations, thus requiring a much bigger science suite and would probably require much longer and heavier instrument booms.

\section{Attitude and Control System, AOCS}

The primary duty of the AOCS is to stabilise the spacecraft prior to and during sail deployment. Should sail deployment fail the sail can be jettisoned and the AOCS can perform it's secondary function and control the spacecraft for a two-year conventional magnetotail mission, in an inertially fixed orbit. We thus note that even a failed sail deployment will allow some mission engineering goals to be attained, through a successful sail jettison and separation manoeuvre. The AOCS uses cold gas when the solar sail is not deployed. We recall that the sail performs post-sail deployment attitude control manoeuvres, thus the AOCS would be largely a redundant system in a successful mission.

\section{Telemetry, Tracking and Command System, TT\&C}

The link budget is defined following analysis of potential communication scenarios, where the link window length is set by the spacecraft / ground-station visibilities. Following a trade of on-board data requirements and on-board RF output power we can better define each. From the detailed sail trajectory data we can analyse the communication opportunities for a range of potential ground-sites to quantify the issues regarding slant range, frequency of window and length of window. The slant range is typically evenly spaced from perigee to apogee, while the time above the horizon (defined as horizon 
plus $10 \mathrm{deg}$ ) typically clusters around $600-800$ minutes. $^{4}$ Furthermore, the time between communication opportunities is typically $1.1-1.2$ days, hence an assumed data latency of $<4$ days allows for three consecutive opportunities to be missed due to ground station unavailability.

A $5 \mathrm{~W}$ S-band solution is adopted using a horn antenna to communicate with a $15 \mathrm{~m}$ ESTRACK station. A ranging capability is included within the link budget analysis, however it is not envisaged that this will be required on a continuous basis. The variation of the communication link rate over the GeoSail operational orbit is shown in Figure 5 for a range of RF powers from 1 - $20 \mathrm{~W}$. Figure 5 also shows the variation in spacecraft mass and sail size over the same range. We note that the sail size varies by just over $1 \mathrm{~m}$ over the considered RF output range and as such we conclude sail size is insensitive to RF output power requirements of less than $20 \mathrm{~W}$. We note that the sensitivity to RF power has a clear step-function relationship. Thus, the impact on sail size is relatively minor if we use $5 \mathrm{~W}$ rather than $1 \mathrm{~W}$, increasing sail size by $\sim 0.17 \mathrm{~m}$, while an increase to $6 \mathrm{~W}$ increases the sail size by a further $0.1 \mathrm{~m}$, it is due to this relative insensitivity that a $5 \mathrm{~W}$ RF output was selected.
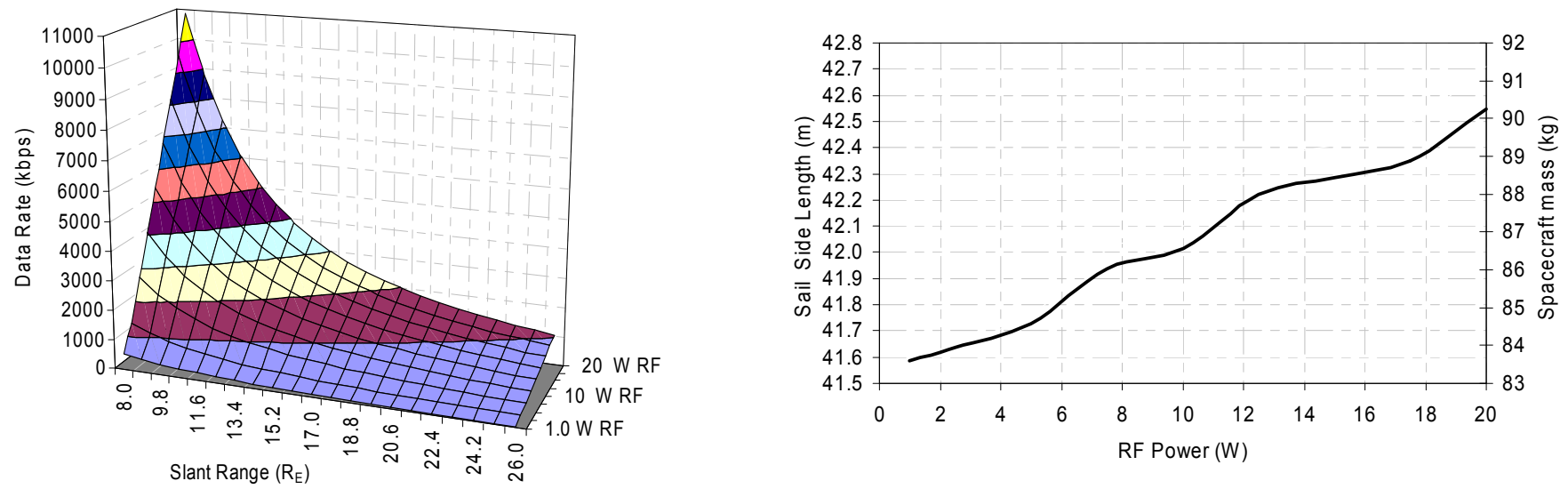

Figure 5 Variation of link budget telemetry capability with slant range and RF power (left) and variation of spacecraft mass and sail side length with RF power. 
The mounting of antennae onto the spacecraft / sail must be carefully designed. The large antenna $3 \mathrm{~dB}$ beamwidth ( $>90 \mathrm{deg}$ ) means that two antenna can provide $\sim 2 \pi$ steradian coverage with redundancy. However, provision of full $4 \pi$ steradian coverage is much more complex due to the large sail surface, which will in effect occlude half of the sky despite the sail film cut-out at the centre of the sail. We also note that reflection of the transmitted signal from the front of the sail could cause destructive interference, although the reflectivity of the sail at radio frequencies is unclear and must thus be investigated. One possible solution would be to mount an antenna in the plane of the sail, such that the sail occludes only a small section of the sky; enabling very close to $4 \pi$ steradian coverage.

\section{On-Board Data Handling, OBDH}

The GeoSail data system is characterised by the continuous acquisition of science and engineering data at a relatively low rate. The data is stored on-board during periods of ground station non-visibility or non-availability for later downlink. The design drivers were primarily simplicity and low cost (fiscal and spacecraft resource), while maintaining as low risk as possible. We note that radiation is not a design consideration; however the thermal environment is of concern due to the prolonged shadow event each orbit. The OBDH system is based on commercial-of-the-shelf (COTS) technology, aided by the low radiation levels anticipated during the mission. The avionics is designed as an Integrated Control Data System, supporting flight software for command and data management functions as well as the attitude control and navigation functions.

The level of required data storage on-board is a function of the TT\&C strategy. As communication link frequency is increased the amount of data stored on board will decrease. Using the TT\&C capabilities in Figure 5 we can define the on-board data storage requirements by varying the communications strategy, along with the data stream acquisition rate. The science data rate stream is $7.672 \mathrm{kbps}$ (at zero 
compression), thus any value over this is available as engineering data collection. Note that while a 2:1 compression is assumed during communication links no compression rate was assumed during the OBDH system analysis, rather it was assumed the data must be stored prior to being compressed. This makes for a suitably conservative design strategy. As this is a demonstration mission we will assume that engineering data requirements are rather high and as such we will consider data stream acquisition rates of $\geq 10 \mathrm{kbps}$. Using the solar sail trajectory information and recalling that we have a minimum data latency requirement of $<4$ days we can vary the minimum downlink length and minimum time between links to optimise ground station use, ensuring against very short links and allowing the ground station to be used by other missions by setting a suitably long minimum time between links.

Figure 6 shows the variation in spacecraft mass and sail side length with the number of 512 Mbyte storage units. We note that the sail side length increases by $1 \mathrm{~m}$ when we go from 2 storage units up to 12 and then by a further metre from 12 units to 17 units, while we recall from Figure 5 that a variation in RF output power from $1 \mathrm{~W}$ to $20 \mathrm{~W}$ increases sail size by only $1 \mathrm{~m}$. Thus, we conclude that at all times we wish to minimise the on-board data storage requirements by maximising the downlink data rate through increasing the RF output power. This comparison is also clear in Figure 6 due to the lower gradient of the data acquisition line. For any given data acquisition rate the minimum required onboard memory storage for a maximum data latency of 4 days, with $50 \%$ margin, can be found. It is thus possible to define the required RF output power for a minimum link duration, frequency and total data acquisition rate to ensure that the maximum data latency requirement is not invalidated. For a minimum frequency communication link of $>3$ days and link length of $>300$ minutes, it is found that we never have more than 4 days between links. A minimum link frequency of 3 days means that we require no more than 2 or 3 links per week, helping to reduce ground support costs. Note also that the maximum link duration is 1175 minutes $(\sim 19.6 \mathrm{hrs})$, thus with a minimum link frequency of 3 days the ground-station will be available for other uses for over 50 hours, worst-case, prior to the next GeoSail 
communications event. Furthermore, it typically does not require a full communications window to download all the data from the spacecraft.

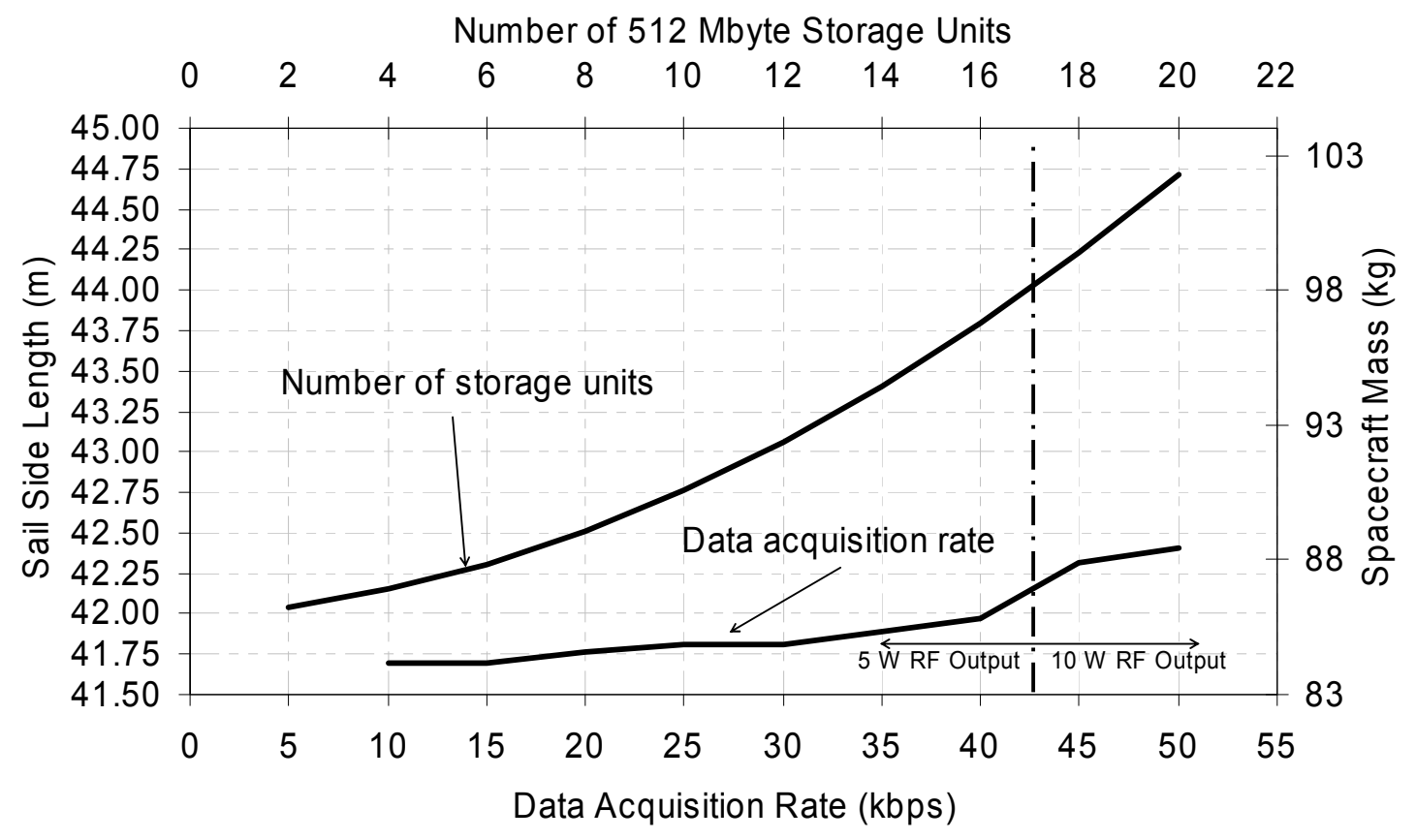

Figure 6 Spacecraft mass and sail side length variation with number of 512 Mbyte storage units and data acquisition rate variation effect on spacecraft mass and sail side.

From Figure 5 we recall that the sail size is fairly insensitive to RF power; however it does have a step function relationship. We thus set RF power to $5 \mathrm{~W}$, the bottom of the first step and check to see if the data latency requirement is met. With a data acquisition rate of $10 \mathrm{kbps}$ it is found that the data storage level does not breach 480 Mbytes and that the 4 day data latency ceiling is also not broken. Maintaining a constant frequency of downlink and minimum downlink duration we can similarly quantify the required RF output level for a range of data stream acquisition rates. It is found that a RF output power of $5 \mathrm{~W}$ is sufficient for data stream acquisition rates up to $40 \mathrm{kbps}$. At greater than 45 kbps the RF output level is increased to $10 \mathrm{~W}$ which is the bottom of the next step up in sail size and has negligible variation in sail size from $7.5 \mathrm{~W}$, but will reduce ground costs notably due to the reduced ground support requirements. It may be preferable to have a RF power above the minimum required 
level defined in this report due to the relative insensitivity in sail size with respect to RF power and the savings in ground costs that can be attained. The effect of data acquisition rate on sail side length is shown in Figure 6, where we note that it is of negligible effect. The GeoSail OBDH system is designed for a total data stream rate of $15 \mathrm{kbps}$, thus the excess downlink capability at $5 \mathrm{~W}$ RF power will reduce ground-station / ground-support costs somewhat.

\section{Thermal and Radiation}

Platform thermal control is required to maintain spacecraft systems within the operating temperature range in all spacecraft mission and operational modes, defined currently as $5^{\circ} \mathrm{C}-25^{\circ} \mathrm{C}$. The defined thermal bounds are conservative in order to maintain an extra level of caution. The thermal design selected for GeoSail uses both passive and active techniques. Due to the large spacecraft / Earth distances the thermal input reflected from Earth has almost no effect on the spacecraft. The maximum sail temperature is $272 \mathrm{~K}$, modelled following Ref. 10. Thus, the sail temperature is less than the required spacecraft operating temperature and will have no input to the thermal flux calculations. Due to the long shadow event each orbit it is not possible to have solely passive thermal control and instead we require heaters to maintain the spacecraft within design bounds. We note that the solar arrays are thermally isolated from the spacecraft structure to minimise the required heater power during the eclipse periods. Furthermore, the batteries are maintained at low temperatures (to increase efficiency) by thermally isolating them from the spacecraft internal environment. In general however, the spacecraft control is maintained not at the element level but instead at the environment level, as for Cluster II. 
A provisional radiation analysis was conducted using the SPENVIS tool, ${ }^{\mathrm{i}}$ allowing us to conclude that for a total shielding thickness of $4 \mathrm{~mm}$ the total ionising dose will be $3.5 \mathrm{krad}(\mathrm{Si})$. We note that typical soft COTS technology fails at $5-10 \mathrm{krad}$, giving a required shielding thickness of $>2 \mathrm{~mm}$. Thus little or no additional structure will be required for radiation shielding and we can classify this as a low radiation mission.

\section{Power}

The main power system design drivers are:

- During launch and systems initiation the power shall be provided by Li-Ion secondary batteries.

- Science operations must continue unhindered through the shadow event each orbit.

- Magnetic cleanliness is required by the science suite.

- Mass and cost should be minimised as much as possible through heritage.

- Solar arrays must be sized for the physical loads during the orbit raising manoeuvre.

Degradation due to radiation is not a design driver, neither is solar array solar aspect angle, which following array deployment will be less than $5 \mathrm{deg}$ at all times due to the GeoSail sail control angle requirements for a zero sail pitch angle. A quasi-regulated power system using direct energy transfer to reduce electromagnetic interference was selected due to its simplicity, the relatively low power requirements and long re-charge period available on each orbit. With solar distance remaining constant over the mission duration, solar input and temperature variations, in daylight, are not large. Thus, a linear shunt regulator can meet the design drivers and be used to improve bus cleanliness.

http://www.spenvis.oma.be 
The End-Of-life (EOL) power load per sub-system in each mode and total EOL power load per mode are detailed in Figure 7. The spacecraft operations are split into six different modes to enable an enhanced analysis of the power requirements, the modes are:

- Launch

From launch minus 3 minutes to spacecraft acquisition plus 10 minutes, all power from batteries. Limited communications capability for engineering data only.

- Hibernation

All systems in stand-by except essential systems, spacecraft automatically exits this mode if defined criteria are met and enters emergency mode.

- Science \& Engineering The nominal operational mode, no communications.

- Communications Science \& Engineering operations mode, with communications link also possible.

- Emergency Mode All non-essential systems in hibernation mode; low-rate communications possible. Spacecraft will automatically enter this mode if defined criteria are met.

- Shadow Science \& Engineering operations continue, no communications other than via emergency mode, all non-vital systems are in stand-by to minimise power load during the long shadow events.

We note that the thermal sub-system is the most power intensive system, due to the requirement to radiate sufficient heat in sunlight and then maintain sufficient heat by running heaters during shadow mode. The communications mode is the most power intensive mode. The power stated for launch mode is the equivalent power for duration of $4 \mathrm{hrs}$, thus the mode EOL power load is $28 \mathrm{~W}$-hrs. The shadow power load is however the design driver for secondary batteries, requiring a battery capacity of $1608 \mathrm{~W}$-hr, for 8 cells in series at $3.35 \mathrm{~V}$ per cell, a cell energy of $10 \mathrm{a}-\mathrm{hr}$ and a $50 \%$ discharge depth, giving a total Li-Ion battery mass of $5 \mathrm{~kg}$. The number of cycles for the batteries is 175 over the core 2-year GeoSail mission. The solar cells are assumed to be Spectrolab 26.8\% Improved Triple Junction 
solar cell arrays, which minimises array mass and volume, while preserving a reasonable array cost with respect to the benefits. The array is split into two wings, which stow against the main structure during launch and deploy by rotating the main gimbal $90 \mathrm{deg}$, then unfolding once.
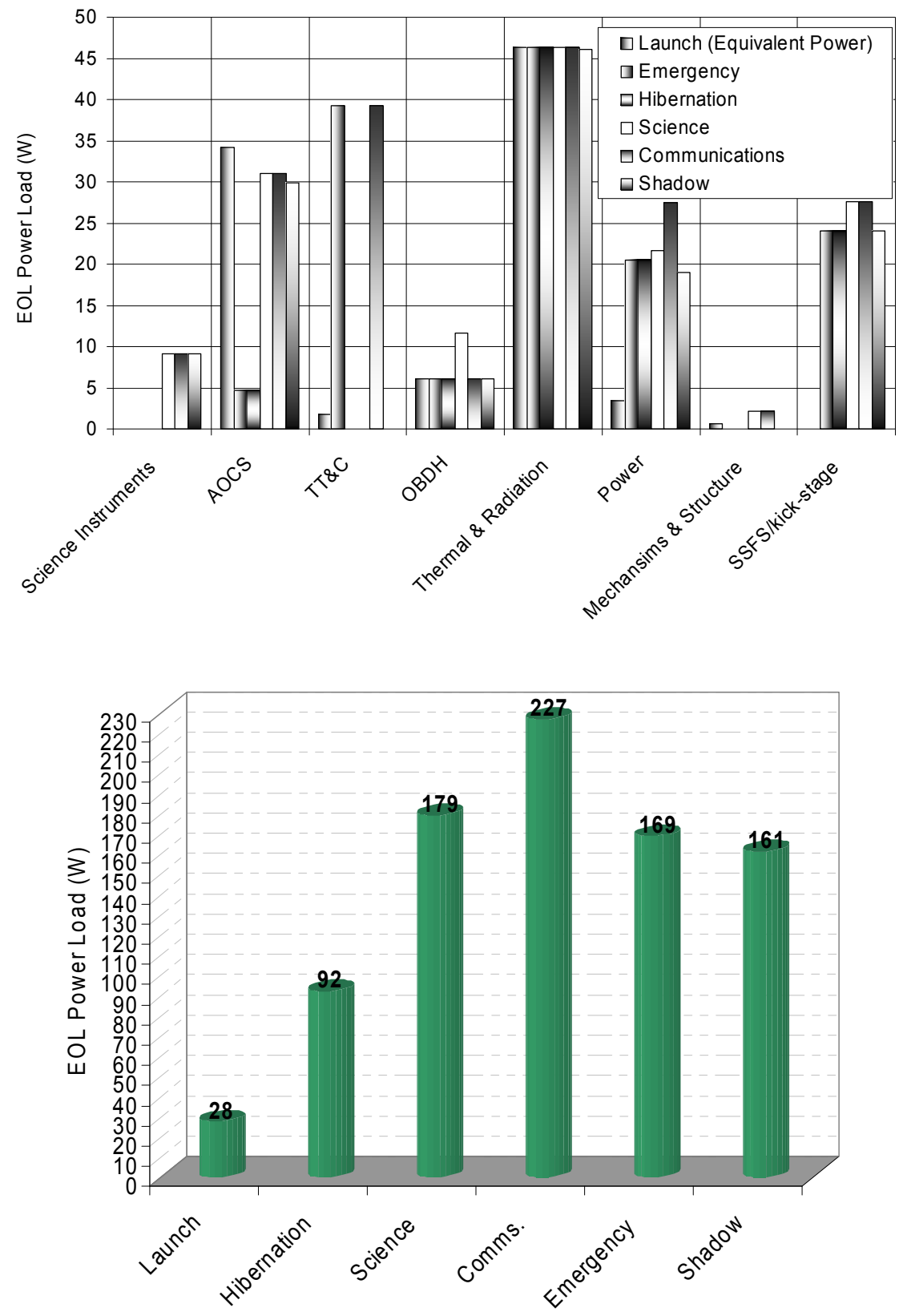

Figure 7 EOL power load per sub-system in each mode (legend corresponds to left-to-right columns per mode) and total EOL power load per mode 


\section{Mechanisms and Structure}

The structural analysis of GeoSail strives to minimise mass, while maintaining as much heritage as possible to minimise cost and risk. The primary structure is composed of carbon-fibre reinforced plastic / Aluminium (CFRP/Al) sandwich panels without heat-pipes, with total thickness $30 \mathrm{~mm}$ and face-sheets of $0.6 \mathrm{~mm}$. Due to the low radiation environment of GeoSail no additional structure is required for radiation protection. The primary structure is a rectangular box of length $1 \mathrm{~m}$ and end width $0.45 \mathrm{~m}$. A secondary structure of aluminium framework is provided to support internal mounting of systems and for load paths during the orbit-raising manoeuvre.

An adaptor is included within the Mechanism and Structure system to allow solar sail jettison, which will be an end-of-life engineering goal, to validate such a concept for later sail missions. The other side of this adaptor is detailed within the solar sail sub-system. The instrument booms are under instrument responsibility, however a mass allocation is included in the system budget.

\section{Kick-Stage Analysis}

The nominal on-orbit mass, see Table 1, must be delivered to the GeoSail operational orbit by the kickstage, prior to sail deployment. The orbit acquisition procedure is different depending on the launch vehicle selected. Assuming a Vega launch we define the kick-stage as in Table 3.

The kick-stage is designed to deliver the spacecraft / sail system into the GeoSail orbit, separate from it and then slowly drift away while the solar sail is deploying. The kick-stage engineering analysis unit allows the kick-stage to perform crude attitude control and station keeping at a safe distance from the sail while it deploys. The system has a laser ranger so as to allow accurate determination of sail - kickstage range. On board the kick-stage engineering analysis unit is an S-band communications suite to 
allow collected data to be returned directly to Earth in the event of a catastrophic failure during sail deployment. This system can be used in conjunction with the sail communication system to validate the sail - kick-stage range. The kick-stage will observe the sail deployment using two wide-angle cameras and two narrow angle cameras, operating mainly in the visible spectrum although it may be of interest to make some observations at other wavelengths. The kick-stage is powered by primary batteries following separation from the main spacecraft and by the solar arrays on the spacecraft while attached. The primary batteries are sized to be $50 \%$ discharged after 24 hours of separated flight. Sail deployment is anticipated to take less than 6 hours.

The insertion $\Delta v$ requirement is $3841.5 \mathrm{~ms}^{-1}$, which in-order to fit within the Vega vehicle must be delivered by a bi-propellant system. The kick-stage uses nitric oxide in nitrogen tetroxide (MON) oxidiser and monomethylhydrazine $(\mathrm{MMH})$ fuel, in a 2.27 mixture ratio, delivering a steady state $\mathrm{I}_{\mathrm{sp}}$ of 320 seconds, based on the S400/2 engine by DASA. The nominal propellant mass for GeoSail orbit acquisition is $837.4 \mathrm{~kg}$, giving a total kick-stage wet mass of $1040.1 \mathrm{~kg}$. The kick-stage system budget elements such as tank mass and volume are sized to include propellant residuals, however propellant residuals are not included within the ESA system level budget and as such are added to the launch mass after this margin has been included, as shown in Table 1. From Table 1 we note that the nominal launch mass is $1186.1 \mathrm{~kg}$, which in addition to a system level margin of $20 \%$ and propellant residuals at $1.5 \%$ propellant mass gives a total launch mass of $1435.9 \mathrm{~kg}$. The total launch mass is $89.1 \mathrm{~kg}$ less than the Vega launch capability to a circular $1500 \mathrm{~km}$ orbit, within the ecliptic plane, giving a launch mass margin of $5.8 \%$. 


\begin{tabular}{|c|c|c|c|c|}
\hline \multicolumn{2}{|l|}{ Component } & $\begin{array}{c}\text { CBE Mass } \\
(\mathrm{kg})\end{array}$ & $\begin{array}{c}\text { DMM } \\
(\%)\end{array}$ & $\begin{array}{c}\text { CBE Mass + DMM } \\
(\mathrm{kg})\end{array}$ \\
\hline \multicolumn{2}{|l|}{ Laser ranger } & 2.5 & 10.0 & 2.8 \\
\hline \multicolumn{2}{|c|}{ Inertial Measurement Unit (IMU) } & 2.8 & 5.0 & 2.9 \\
\hline \multicolumn{2}{|l|}{ Earth Sensor } & 0.6 & 5.0 & 0.6 \\
\hline \multicolumn{2}{|c|}{ Cold gas control system (at $5 \%$ of kick-stage mass) } & 9.6 & 5.0 & 10.1 \\
\hline \multicolumn{2}{|l|}{ Waveguide Horn Antenna } & 1.2 & 5.0 & 1.3 \\
\hline \multicolumn{2}{|c|}{ Small Deep Space Transponder (SDST) } & 4.5 & 5.0 & 4.7 \\
\hline \multicolumn{2}{|c|}{ Solid State Power Amplifier (RF output $=5 \mathrm{~W}$ ) } & 2.5 & 5.0 & 2.6 \\
\hline \multicolumn{2}{|l|}{ RF Distribution Unit } & 3.6 & 5.0 & 3.8 \\
\hline \multicolumn{2}{|l|}{ Additional TT\&C Hardware } & 0.7 & 5.0 & 0.7 \\
\hline \multicolumn{2}{|c|}{ Lithium Sulphur Dioxide ( $\mathrm{Li}^{-\mathrm{SO}_{2}}$ ) Cells (96 of) } & 11.0 & 5.0 & 11.6 \\
\hline \multicolumn{2}{|l|}{ Primary Structure } & 4.0 & 5.0 & 4.2 \\
\hline \multicolumn{2}{|l|}{ Secondary Structure } & 1.7 & 5.0 & 1.8 \\
\hline \multicolumn{2}{|l|}{ Wide Angle Camera } & 2.0 & 10.0 & 2.2 \\
\hline \multicolumn{2}{|l|}{ Narrow Angle Camera } & 1.2 & 10.0 & 1.3 \\
\hline \multicolumn{2}{|c|}{ Kick-Stage Engineering Analysis Unit sub-total } & 47.9 & 6.1 & 50.6 \\
\hline \multicolumn{2}{|l|}{ Thrusters } & 6.8 & 5.0 & 7.1 \\
\hline Valves, pipes \& other devices & $30 \%$ of tank mass & 25.9 & 5.0 & 27.2 \\
\hline Fuel Tank & $10 \%$ of fuel mass & 26.4 & 5.0 & 27.7 \\
\hline Oxidiser Tank & $10 \%$ of oxidiser mass & 59.9 & 5.0 & 62.9 \\
\hline Pressurant Tank & $30 \%$ total tank mass & 25.9 & 5.0 & 27.2 \\
\hline Total & & 192.7 & 5.2 & 202.7 \\
\hline
\end{tabular}

Table 3 Kick-stage system, with Engineering Analysis Unit mass breakdown

SEP Systems Analysis

Following the SEP mission analysis presented earlier we define a SEP system following the Qinetiq T5, variable specific impulse, analysis for a 2 year mission. Recall that a SEP propulsion system (assuming Qinetiq T5 thrusters) will likely be favourable over a solar sail propulsion system if the 
nominal on-orbit dry mass is between $100 \mathrm{~kg}$ and $150 \mathrm{~kg}$. The SEP system is detailed in Table 4. Note the spacecraft remains 3-axis stabilised to maintain consistency. A spinning spacecraft would probably be preferable for all propulsion systems; however it is unlikely any propulsion system would be more or less compatible with such a configuration.

SEP systems are characterised as low reaction mass, high power systems. Thus, while high-energy missions are often enabled by electric propulsion these missions tend to be high mass due to the high power requirements. A recent study attempting to integrate an ion propulsion system into a low mass spacecraft found that the spacecraft dry mass could not be reduced much below $182 \mathrm{~kg}$, including system level margin. ${ }^{23}$ The primary variations between the solar sail and SEP systems analysis is the power and structure mass allocations. The Qinetiq T5 thrusters draw a maximum power of $677 \mathrm{~W}$, plus margin, giving a maximum power load design case of $1296 \mathrm{~W}$, considerably greater than the maximum power load design case of $182 \mathrm{~W}$ for the solar sail mission. As a result of the increased power load the solar array area increases from $0.8 \mathrm{~m}^{2}$ up to $5.3 \mathrm{~m}^{2}$, giving a mass growth from just under $5 \mathrm{~kg}$ to just under $30 \mathrm{~kg}$. Similarly, the battery mass along with the power distribution and conditioning units, and the harness mass all grow significantly. As a result of the increased power system mass the thermal environment within the spacecraft is altered significantly. This is handled primarily through slightly extra passive thermal control and an increase in spacecraft internal volume, resulting in a structural mass increase. Furthermore, the large solar arrays also require larger solar array drive assemblies and a re-enforced structure to support the arrays. The SEP system is detailed in Table 4, the system is fully redundant, including thrusters. Sizing the kick-stage, system margins and propellant residuals we find that the total launch mass is $1736 \mathrm{~kg}$, for a Vega launch. Therefore, a Vega cannot launch the stack and we must consider a Soyuz vehicle instead. The kick-stage uses a solid motor, giving a launch mass margin of $83 \%$. We note that since a solid motor cannot be restarted the AOCS would need to be augmented to perform any orbit insertion correction manoeuvre. 
The increase in launch vehicle capability significantly reduces launch mass, however it also significantly increases orbit acquisition cost, defined as launcher plus kick-stage.

\section{Chemical Propulsion System Analysis}

Following the earlier mission analysis presented for chemical propulsion it is clear that of the currently mature propulsion technology options SEP is the better quality solution. Using engineering judgement we can rapidly generate an assessment of the chemical propulsion systems analysis. The spacecraft mass minus primary propulsion system will likely be only marginally heavier than the solar sail spacecraft, with an increased mass requirement for the structure depending on the selected thrust value of the primary propulsion system. We thus estimate the spacecraft mass minus primary propulsion system as $95 \mathrm{~kg}$. The primary bi-propellant propulsion system, minus tanks and valves, can be estimated at $10 \mathrm{~kg}$, we thus estimate a spacecraft dry mass minus tanks, pipes and values of $105 \mathrm{~kg}$. Recall that the $\Delta v$ per year is $2273.4 \mathrm{~ms}^{-1}$, plus finite burn losses at $5 \%$, giving a yearly $\Delta v$ requirement of $2387.1 \mathrm{~ms}^{-1}$. Assuming an $\mathrm{I}_{\mathrm{sp}}$ of 350 secs we can thus estimate the propellant, tank, valves and piping masses, giving a nominal on-orbit mass of $658 \mathrm{~kg}$. Note that the nominal on-orbit mass includes finite burn losses, but not propellant residuals. As with the SEP analysis, we require a Soyuz-2B launch rather than the Vega. The Soyuz-2B launch mass is just over $600 \mathrm{~kg}$, including propellant residuals on the kick-stage only. The Soyuz-2B launch mass margin is $1618.8 \mathrm{~kg}$. 
System

CBE Mass DMM CBE Mass + DMM

\begin{tabular}{|c|c|c|c|}
\hline & $(\mathrm{kg})$ & $(\%)$ & $(\mathrm{kg})$ \\
\hline Science Instruments & 11.5 & 10.0 & 12.7 \\
\hline Attitude \& Orbit Control System, AOCS (dry) & 10.5 & 5.0 & 11.0 \\
\hline Telemetry, Tracking and Command, TT\&C & 16.3 & 5.0 & 17.1 \\
\hline On-Board Data Handling, OBDH & 1.1 & 10.0 & 1.2 \\
\hline Thermal \& Radiation & 1.9 & 10.0 & 5.4 \\
\hline Power & 64.6 & 10.0 & 71.1 \\
\hline Mechanisms \& Structure & 32.8 & 8.3 & 36.4 \\
\hline Spacecraft Nominal Dry Mass At Launch & & & 154.8 \\
\hline AOCS propellant, inc. a margin & & & 2.3 \\
\hline Spacecraft Nominal Wet Mass at Launch & & & 157.0 \\
\hline SEP System Nominal Mass at Launch & 33.7 & 14.7 & 38.8 \\
\hline Nominal On-Orbit Dry Mass & & & 195.8 \\
\hline Xenon Fuel & 38.7 & 14.7 & 44.4 \\
\hline Nominal On-Orbit Wet Mass & & & 240.2 \\
\hline Kick-stage mass (dry) & 12.6 & 5.0 & 13.2 \\
\hline Solid Propellant & & & 103.6 \\
\hline Kick-stage mass (wet) & & & 116.8 \\
\hline Nominal Launch Mass & & & 312.6 \\
\hline ESA System Level Margin & & $20 \%$ & 62.5 \\
\hline \multicolumn{2}{|c|}{ Residual Xenon fuel (at $1.5 \%$ of Propellant Mass) } & & 0.6 \\
\hline \multicolumn{2}{|l|}{ Solid Propellant Residual } & & 1.5 \\
\hline \multicolumn{2}{|l|}{ Total Mass At Launch } & & 377.2 \\
\hline \multicolumn{2}{|l|}{ Soyuz-2b launch capacity to 23 Earth radii apogee } & & 2220.0 \\
\hline Launch Margin & & & $1842.8(83.0 \%)$ \\
\hline
\end{tabular}

Table 4 SEP system overview 
Visualisation and Volumetric Analysis

Following the mission and systems analysis studies an outline visualisation and volumetric analysis of the GeoSail concept is presented, ensuring that the spacecraft, sail and kick-stage are correctly defined and can be launched within the volumetric constraints of the Vega fairing. The launch stack configuration is shown in the Vega fairing in Figure 8. We note that the GeoSail stack fits easily within the available volume. Note that until the solar arrays are deployed the spacecraft is reliant on battery power alone.

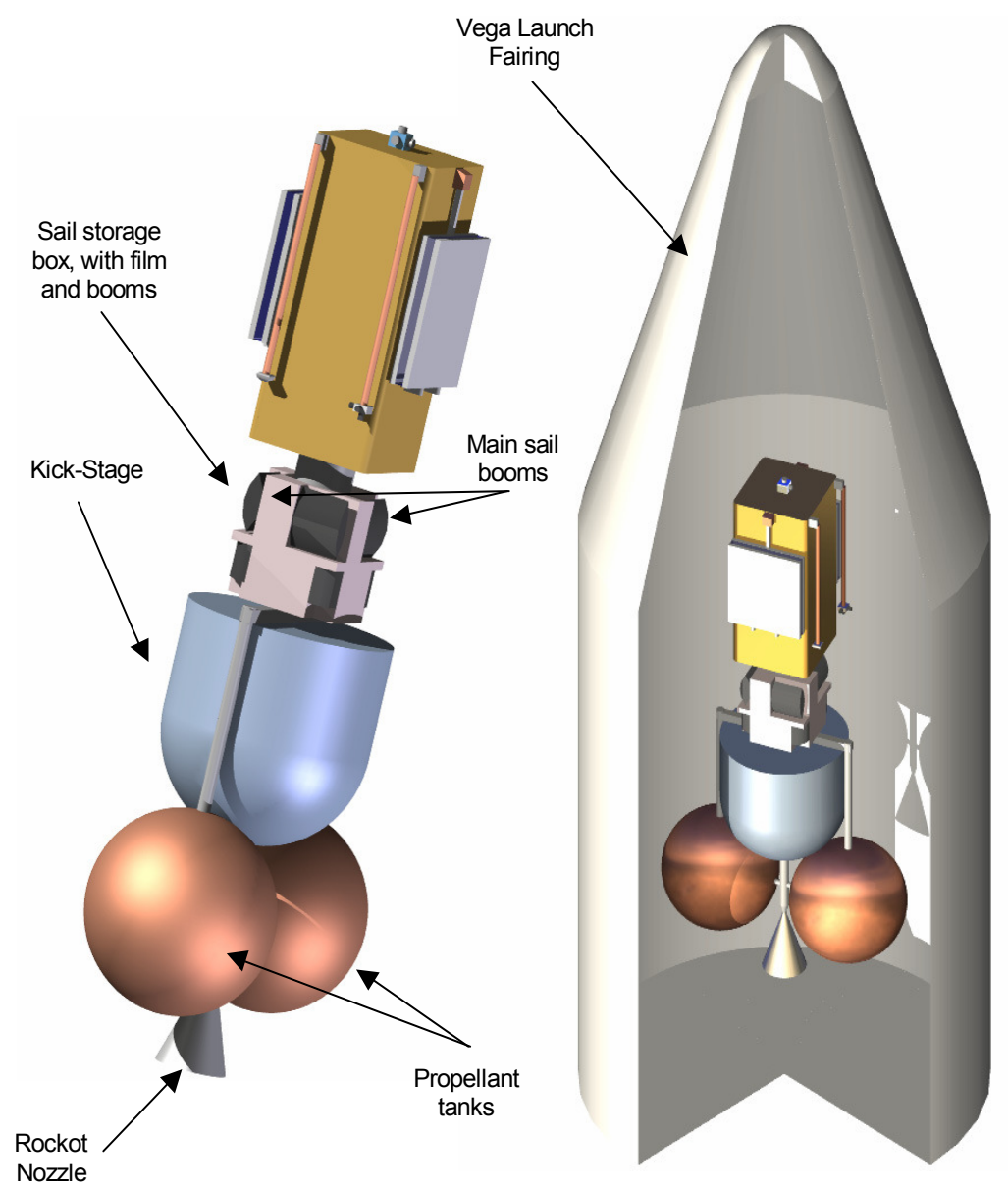

Figure 8 GeoSail launch stack and in Vega fairing

\section{Solar Sail System Analysis and Configuration}

The sail is at all times Sun-pointing, that is to say, at zero deg pitch angle with respect to the Sun-line. Therefore, the sail attitude control requirements for GeoSail are low, as a fixed zero pitch setting can be 
maintained largely through passive means. However, it is envisaged that the sail will maintain attitude control and pointing for the science suite, thus some attitude error correction capability will be required to maintain the science suite within its pointing requirements. It was demonstrated in Ref. 6 that the SPO mission has a slew rate capability requirement of $10 \mathrm{deg} / \mathrm{day}$. GeoSail should demonstrate the technology requirements of future, mid-term, solar sail missions. Therefore, the GeoSail spacecraft should be capable of sail slew rates of order $10 \mathrm{deg} /$ day and should at some point within the mission demonstrate this capability, as for example in Ref. 13. The sail systems are sized and configured to meet this requirement.

The large moment of inertia of a solar sail and the low-frequency structural dynamics present many unique attitude control challenges for the solar sail designer. It is beyond the scope of this paper to examine and attempt to determine the optimal attitude control solution. Following the discussion in Ref. 6 and 21, we define a dual attitude control system, which exclusively uses solar radiation. The use of $\mu$ PPT thrusters on the sail boom tips was not considered at this stage. The selected dual attitude control system is based on a central deployed and gimballed boom working in co-operation with small tip-vanes. When the sail film is deployed it is unlikely that the film will deploy in an exactly symmetrical fashion, thus a centre-of-pressure / centre-of-mass (CoP / CoM) offset error appears inevitable. An offset error of $0.25 \%$ of the sail side length has been determined to be a likely error value. ${ }^{6}$ Within the GeoSail mission it is critical that the science suite be as far removed from the solar sail as possible, thus the concept of mounting the spacecraft on a large central boom is attractive. In order to overcome the difficulties previously outlined with the use of a central boom ${ }^{21}$ it was decided that the boom will have only a limited control authority, steps of, say, 0.1 deg through a ratchet system. The boom will be capable of moving in two-degrees of freedom. The primary function of the central boom is to perform the gross error correction of the CoP / CoM error, thus we envisage the boom will only be moved very infrequently throughout the mission, say, once every 6 months to compensate for 
sail reflective surface degradation and performance variation of individual sail quadrants. The primary function of the tip-vanes is thus simply to perform attitude control manoeuvres and correct for the remaining very small $\mathrm{CoP} / \mathrm{CoM}$ error which could not be corrected by the central boom due to its limited control authority. The tip-vanes are sized to be fully redundant, thus both the roll and both the pitch vanes are the same size.

The method of sail deployment is critical to mission success. The optimal method will only become clear following a detailed trade however several key points are noted at this stage of analysis. During deployment the spacecraft attitude must be maintained by the AOCS, thus it is important to maintain as a low moment of inertia as possible in order to minimise this systems mass and volume. To this end if the sail booms are deployed prior to the sail film, attitude control could be difficult to maintain if only the centrally mounted AOCS is available. Therefore, it would seem prudent to deploy the sail film at the same time as the sail booms, thus the sail film should allow for near-passive attitude control assuming a quasi-uniform and controlled deployment at zero pitch to the Sun-line. Similarly, if the centrally mounted spacecraft boom is deployed prior to the sail then attitude control could be difficult to maintain during the sail deployment, however if it is not deployed until after the sail then attitude control could be difficult to maintain following deployment. If the centrally mounted spacecraft boom can be deployed after the sail film this would provide an excellent opportunity to analyse the effect of the sail on the spacecraft science instruments, which could analyse the local environment close to the sail and then over a range of distances until the boom is fully deployed. It thus appears that perhaps the best science and engineering based approach would be to deploy the centrally mounted spacecraft boom after the sail film and booms have been simultaneously deployed. 


\section{Solar Sail Systems Analysis}

The effect of varying the sail technology level on sail size, mass and launch mass was considered. Note that as the sail technology level is varied, and hence sail size varied, the mass of the spacecraft varies slightly, since the sail is essentially another spacecraft sub-system. The sensitivity analysis is performed for CP-1 (clear plastic) and Polyethylene Terephthalate (PET) film, as these two films represent the most and least dense candidate substrates, respectively. We note that varying the sail substrate material has negligible effect and thus selection must be based on cost, structural, thermal and radiation properties of each candidate material. It was found that the main sail boom specific mass has negligible effect on the sail size, typically varying the boom length by less than $2 \mathrm{~m}$ for a change in specific mass of $100 \mathrm{gm}^{-1}$. The variation in boom specific mass has a more pronounced effect on sail system mass and thus launch mass. In order to remain within the launch mass limit of the Vega launcher we must reduce boom specific mass below $75 \mathrm{gm}^{-1}, 115 \mathrm{gm}^{-1}$, and $160 \mathrm{gm}^{-1}$ for sail film thicknesses of $1 \mu \mathrm{m}, 3 \mu \mathrm{m}$ and $5 \mu \mathrm{m}$. Recall that the use of a Vega launcher is one of the key advantages a sail mission has over either SEP or chemical propulsion missions, hence these limits can be considered hard limits for sail technology assuming the kick-stage technology remains constant.

The effect of varying the sail film thickness on the sail size and launch mass is shown in Figure 9. Sail film thickness has negligible effect on sail size; typically varying the sail boom length by $0.4-0.5 \mathrm{~m}$ for every $1 \mu \mathrm{m}$ variation in thickness. Once again, the launch mass varies more notably than sail size. To remain within the Vega launch mass window we must utilise a sail film thickness of less than $4 \mu \mathrm{m}$ and $6 \mu \mathrm{m}$, depending on boom specific mass. Once again, these limits can be considered hard limits for sail technology assuming the kick-stage technology remains constant.

The effect of varying the mass of the spacecraft attached to the solar sail on the sail size and launch mass was also considered. It was found that the sail boom length varies by $2-3 \mathrm{~m}$ for every $10 \mathrm{~kg}$ of 
spacecraft. Thus, the attached spacecraft mass is the single largest driver of sail size within the GeoSail mission, rather than the sail technology level. We note that the use of a large spacecraft $(200 \mathrm{~kg})$ and a low level of sail technology drives the sail boom length up to approximately $43 \mathrm{~m}$, side length approximately $61 \mathrm{~m}$, which remains within the realm of a demonstration mission. The spacecraft mass must be less than $84 \mathrm{~kg}\left(100 \mathrm{gm}^{-1}\right.$ booms and $5 \mu \mathrm{m}$ film) to $103 \mathrm{~kg}\left(50 \mathrm{gm}^{-1}\right.$ booms and $1 \mu \mathrm{m}$ film), depending on sail technology level in order to fit within the Vega launcher. Once again, this limit can be considered a hard limit for sail technology assuming the kick-stage technology remains constant.

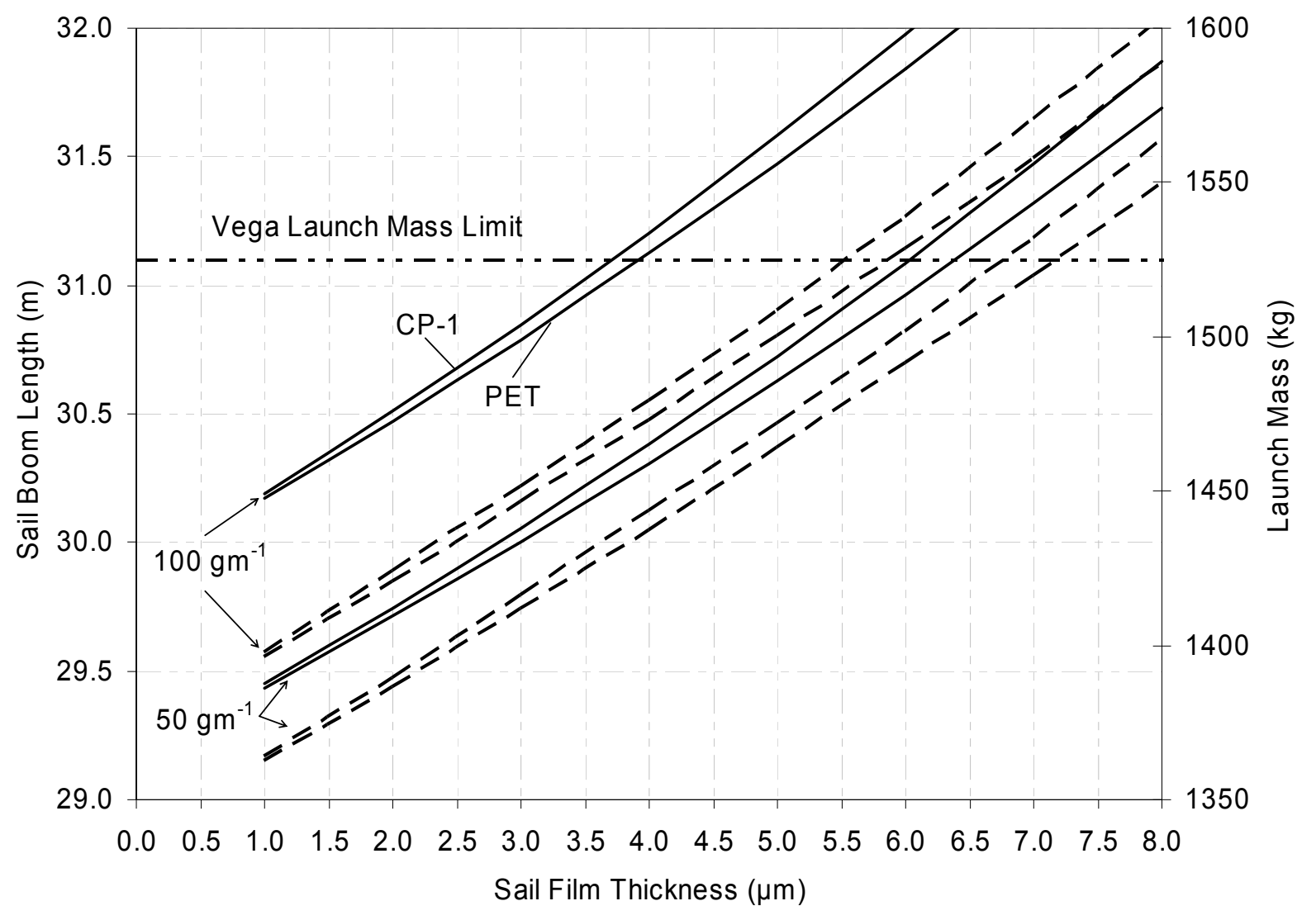

Figure 9 Sail technology level sensitivity analysis, solid lines are launch masses and dashed lines are sail boom lengths, each pair of lines is CP-1 film and PET film as shown for the upper most curve. 


\section{Solar Sail System Definition}

Using the trades presented above we can define the nominal GeoSail solar sail budget, as shown in Table 5. The definition of required technology for the GeoSail mission cannot be performed in isolation, due to the relative insensitivity of sail size to technology used. The required technology is defined only after consideration of the requirements of future sail missions, thus placing GeoSail at the beginning of a carefully planned and thought-out solar sailing roadmap. ${ }^{5}$ It is apparent that the first mid-term solar sail mission will likely be the SPO mission which therefore forms the target of our heritage goals. The SPO requires a minimum boom technology of $100 \mathrm{gm}^{-1}$ at length $111 \mathrm{~m}$ and film thickness of less than $3 \mu \mathrm{m}$, but preferably a boom technology of $65 \mathrm{gm}^{-1}$ at length $108 \mathrm{~m}$ and film thickness of $2 \mu \mathrm{m} .6$ To meet such an equivalent requirement on a $40 \mathrm{~m}$ class solar sail a boom technology of order $40 \mathrm{gm}^{-1}$ is required. Considering GeoSail as part of a near-term solar sailing roadmap, leading towards the SPO mission we see in Table 5 that a sail film of $3.5 \mu \mathrm{m}$ Teonex $^{\circledR}$ is defined for the GeoSail mission. The defined boom specific mass is $40 \mathrm{gm}^{-1}$, significantly lower than required if we consider the mission in isolation. However, this technology level provides a smooth transition from GeoSail through towards the SPO, ${ }^{5}$ though it is unlikely that GeoSail alone will provide sufficient heritage towards selecting a future $150 \mathrm{~m}$ square solar sail mission. Note, the "Engineering analysis suite" in Table 5 consists of items such as stress and toque gauges to monitor the sails physical state during deployment and once it is in operation. Recall, the deployment of the sail will be also monitored by the Kick-Stage Engineering Analysis Unit as detailed in Table 3. The sail system has an assembly loading of $34 \mathrm{gm}^{-1}$, with a sail loading of $84.5 \mathrm{gm}^{-1}$. The defined sail size is $42.8 \mathrm{~m}$ giving a total sail system mass of $59 \mathrm{~kg}$. 


\begin{tabular}{|c|c|c|c|}
\hline System & $\begin{array}{c}\text { CBE Mass } \\
(\mathrm{kg})\end{array}$ & $\begin{array}{c}\text { DMM } \\
(\%)\end{array}$ & $\begin{array}{c}\text { CBE Mass + DMM } \\
(\mathrm{kg})\end{array}$ \\
\hline $3.5 \mu \mathrm{m}$ Teonex ${ }^{\circledR}(\mathrm{PEN})$ & 8.4 & 10.0 & 9.3 \\
\hline $0.1 \mu \mathrm{m} \mathrm{Al} / 0.01 \mu \mathrm{m} \mathrm{Cr}$ (front / rear coating) & 0.59 & 5.0 & 0.62 \\
\hline Main sail bonding & 0.9 & 10 & 1.0 \\
\hline Main sail booms, at $40 \mathrm{gm}^{-1}$ & 4.8 & 20.0 & 5.8 \\
\hline Tip Vanes & 0.02 & 9.0 & 0.04 \\
\hline Tip vane gimbal and motor & 4.0 & 10.0 & 4.4 \\
\hline Harness \& tip vane cabling & 4.5 & 10.0 & 4.9 \\
\hline Central deployable boom, $10 \mathrm{~m}$ length & 5.0 & 20.0 & 6.0 \\
\hline Stowage / Primary structure & 5.3 & 10.0 & 5.8 \\
\hline Secondary structure & 2.1 & 10.0 & 2.3 \\
\hline Instrumentation, electronics, et cetera & 2.5 & 10.0 & 2.8 \\
\hline Engineering Analysis suite & 2.0 & 10.0 & 2.2 \\
\hline Structure for Deployable Boom Housing & 2.0 & 15.0 & 2.3 \\
\hline Deployable Boom Articulation Motor & 4.0 & 15.0 & 4.6 \\
\hline Spacecraft adaptor - carrier side & 3.3 & 10.0 & 3.6 \\
\hline Miscellaneous components & 1.3 & 5.0 & 1.4 \\
\hline Launch vehicle adaptor, spacecraft side & 1.8 & 5.0 & 1.9 \\
\hline Total & 52.5 & 10.0 & 58.9 \\
\hline
\end{tabular}

Table 5 GeoSail solar sail design.

\section{Conclusions}

A detailed study of GeoSail as a solar sail technology demonstration mission in the context of a potential component of the SMART programme has been presented. In this framework, the engineering goals will take slightly greater precedence over the science goals, although a technology demonstration mission that can also provide a novel and useful science return is clearly attractive. In addition to satisfactory sail deployment and operation, the mission concept has been defined to 
demonstrate technologies for safe sail jettison in future missions and potential sail scalability, in addition to an investigation of the sail interaction with the science payload.

A detailed trade-off was presented as to the effect of a variety of parameters on the sail size and launch mass, for example the effect of sail film thickness or the amount of data storage capability on-board the spacecraft. Following these trades a $43 \times 43 \mathrm{~m}$ square solar sail was defined, based on near-term technologies, with a projection towards future solar sail mission requirements. The sail has an assembly loading of $34 \mathrm{~g} \mathrm{~m}^{-2}$, using $3.5 \mu \mathrm{m}$ Teonex ${ }^{\circledR}$ film and a boom specific mass of $40 \mathrm{gm}^{-1}$. The spacecraft mass is $87 \mathrm{~kg}$ and the sail assembly mass is $59 \mathrm{~kg}$, which provides the necessary acceleration of $0.1 \mathrm{~mm} \mathrm{~s}^{-2}$ for Sun-synchronous apse-line precession. When GeoSail is attached to the $1040 \mathrm{~kg}$ kick-stage for transfer, the total launch mass is $1436 \mathrm{~kg}$, which includes a $20 \%$ system margin. This enables the use of a Vega launch vehicle with a launch margin of 5.8\%.

The performance of the solar sail concept (GeoSail) was compared with solar electric propulsion (GeoSEP) and chemical propulsion concepts. It was found that solar sailing significantly outperforms the other propulsion modes in terms of reduced launch mass and hence cost. The reduced launch mass cost was the primary benefit of the solar sail concept over the other propulsion options. GeoSail is a logical choice for a first operational solar sail mission and is currently the only such concept that is truly enabled by solar sail propulsion.

\section{Acknowledgments}

This study was conducted under contract "ESTEC 16534/02/NL/NR: Technical Assistance in the Study of Science Payloads Transported Through Solar Sailing". 


\section{References}

1 Macdonald M., MInnes C. R., "GeoSail; An Enhanced Magnetosphere Mission, Using a Small Low Cost Solar Sail", IAF-00-W.1.06, Proceedings of $51^{\text {st }}$ International Astronautical Congress, Rio de Janeiro, Brazil, 2-6 October 2000.

2 M'Innes C.R., Macdonald M., Angelopolous V., Alexander D., "GeoSail: Exploring the Geomagnetic Tail Using a Small Solar Sail”, J. Spacecraft and Rockets, Vol. 38, No. 4, 2001, pp. 622629.

3 Alexander D., Sandman A. W., Minnes C. R., Macdonald M., Ayon J., Murphy N. and Angelopoulos V., “GeoSail: A Novel Magnetospheric Space Mission Utilizing Solar Sails”, IAC-02IAA.11.1.04, Electronic Proceedings of the $53^{\text {rd }}$ International Astronautical Congress, Houston Texas, 10-19 October 2002.

4 Macdonald M., MInnes C. R., Alexander D., Sandman A., "GeoSail: Exploring the Magnetosphere Using a Low-Cost Solar Sail", Electronic Proceedings of Fifth IAA International Conference on Low-Cost Planetary Missions, ESA Special Publication SP-542, September 2003, pp. 341-349.

5 Macdonald M., MInnes C. R., “A Near-Term Roadmap for Solar Sailing”, IAC-04-U.1.09, Electronic Proceedings of $55^{\text {th }}$ International Astronautical Congress, Vancouver, Canada, 4-8 October 2004.

6 Macdonald M., Hughes G., MInnes C. R., Lyngvi A., Falkner P., Atzei A., "The Solar Polar Orbiter: A Solar Sail Technology Reference Study”, Journal Spacecraft \& Rockets. (In Press)

7 Lyngvi A., Falkner P., Kemble S., Leipold M., Peacock A., "The interstellar heliopause Probe”, Acta Astronautica, Vol. 57, Issues 2-8, July-October 2005, pp. 104-111 
Alexander D., MInnes C. R., Angelopoulos V., Sandman A. W., Macdonald M., "GeoSail: A Novel Solar Sail Mission Concept for Geospace", Proceedings of Space Technology and Applications International Forum (STAIF 2002), Albuquerque, New Mexico, U.S.A., 3 - 7 Feb. 2002.

9 West J., Derbes B., "Solar Sail Vehicle Design For The Geostorm Warning Mission", AIAA2000-5326, Proceedings of the $41^{\text {st }}$ AIAA Structures, Structural Dynamics and Materials Conference and Adaptive Structures Forum, 2000.

$10 \quad$ M Innes C.R., "Solar Sailing: Technology, Dynamics and Mission Applications", SpringerVerlag, London, 1999.

11 Dormand, J.R., Price, P.J., "A Family of Embedded Runge-Kutta Formulae”, Journal of Computing and Applied Mathematics, Vol. 6, 1980, pp $19-26$.

12 Macdonald M., MInnes C. R., "Analytic Control Laws for Near-Optimal Geocentric Solar Sail Transfers", AAS 01-472, Advances in the Astronautical Sciences, Vol. 109, No. 3, 2001, pp. 23932413.

13 Macdonald M., MInnes C. R., "Analytical Control Laws for Planet-Centred Solar Sailing”, Journal of Guidance, Control, and Dynamics, Vol. 28, No. 5, 2005, pp. 1038-1048.

14 Burt E.G.C., "On Space Manoeuvres with Continuos Thrust”, J. Space Science, Vol.15, 1967, pp103-122.

15 Burt E. G. C., "The Dynamics of Low-Thrust Spacecraft Manoeuvres," Journal of the Royal Aeronautical Society, Vol. 72, No. 695, 1968, pp. 925-940.

16 Fearn D. G., "The Design of a Versatile Ion Thruster for Large Communications Spacecraft," IAF-97-S.3.01, $48^{\text {th }}$ International Astronautical Congress, Turin, Italy, October 6-10, 1997.

17 Fearn D. G., Smith P., “A Review of UK Ion Propulsion - a Maturing Technology,” IAF-98S.4.01, $49^{\text {th }}$ International Astronautical Congress, Melbourne, Australia, September 28 - October 2, 1998. 
Fearn D. G., "A Study of the Throttling of the T5 ion Thruster," AIAA 96-3288, 32 ${ }^{\text {nd }}$ AIAA/ASME/SAE/ASEE Joint Propulsion Conference, Lake Buena Vista, Florida, July, 1996.

19 Macdonald M., M'Innes C. R., “Analytic Control Laws for Near-Optimal Geocentric Solar Sail Transfers”, AAS 01-472, Advances in the Astronautical Sciences, Vol. 109, No. 3, 2001, pp. 23932413.

20 Wie B., "Sail Flatness, Attitude, and Orbit Control Issues for an ST-7 Solar Sail Spacecraft", NASA SSTWG FY01, Solar Sail Technical Interchange Meeting, NASA Goddard Space Flight Center, 2001.

21 Murphy D., Wie B., "Robust Thrust Control Authority for a Scalable Sailcraft", AAS 04-285, $14^{\text {th }}$ AAS/AIA Space Flight Mechanics Conference, Maui, Hawaii, 2004.

22 Garrett H., Wang J., "Simulations of Solar Wind Plasma Flow Around A Simple Solar Sail", Proceedings of the $8^{\text {th }}$ Spacecraft Charging Technology Conference, Ed. R. Suggs, MSFC, Huntsville, Alabama, 20 - 24 October 2003.

23 Clark S. D., Fearn D. G., "The Impact of Advanced Platform and Ion Propulsion Technologies on Small, Low-Cost Interplanetary Spacecraft," Proceedings of $5^{\text {th }}$ IAA International Conference of Low-Cost Planetary Missions, ESTEC, Noordwijk, The Netherlands, 24-26 September, ESA SP-542, 2003, pp. 279-288. 